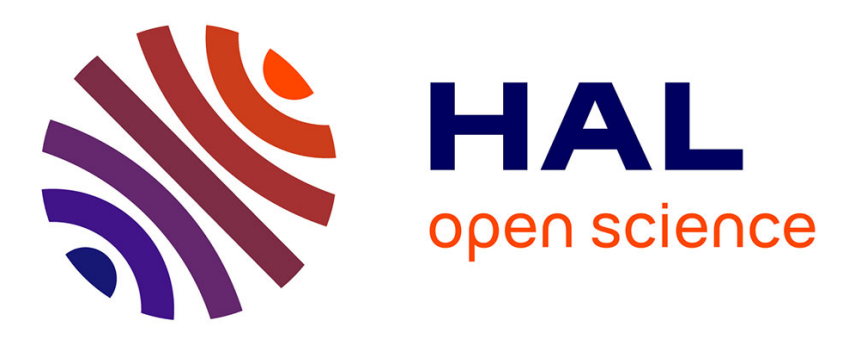

\title{
Tracing the simulated high-redshift circum-galactic medium with Lyman $\alpha$ emission
}

Peter D Mitchell, Jérémy Blaizot, Corentin Cadiou, Yohan Dubois, Joakim A Rosdahl, Thibault Garel

\section{- To cite this version:}

Peter D Mitchell, Jérémy Blaizot, Corentin Cadiou, Yohan Dubois, Joakim A Rosdahl, et al.. Tracing the simulated high-redshift circum-galactic medium with Lyman $\alpha$ emission. Monthly Notices of the Royal Astronomical Society, 2021, 501, pp.5757 - 5775. 10.1093/mnras/stab035 . hal-03444448

HAL Id: hal-03444448

https://cnrs.hal.science/hal-03444448

Submitted on 23 Nov 2021

HAL is a multi-disciplinary open access archive for the deposit and dissemination of scientific research documents, whether they are published or not. The documents may come from teaching and research institutions in France or abroad, or from public or private research centers.
L'archive ouverte pluridisciplinaire HAL, est destinée au dépôt et à la diffusion de documents scientifiques de niveau recherche, publiés ou non, émanant des établissements d'enseignement et de recherche français ou étrangers, des laboratoires publics ou privés. 


\title{
Tracing the simulated high-redshift circum-galactic medium with Lyman $\alpha$ emission
}

\author{
Peter D. Mitchell ${ }^{\star 1}$, Jérémy Blaizot ${ }^{2}$, Corentin Cadiou ${ }^{3}$, Yohan Dubois ${ }^{4}$, \\ Thibault Garel ${ }^{5,2}$, and Joakim Rosdahl ${ }^{2}$ \\ ${ }^{1}$ Leiden Observatory, Leiden University, P.O. Box 9513, 2300 RA Leiden, the Netherlands \\ ${ }^{2}$ Univ Lyon, Univ Lyon1, Ens de Lyon, CNRS, Centre de Recherche Astrophysique de Lyon UMR5574, F-69230, Saint-Genis-Laval, France \\ ${ }^{3}$ Department of Physics and Astronomy, University College London, Gower Street, London WC1E 6BT, UK \\ ${ }^{4}$ Institut d'Astrophysique de Paris, Sorbonne Université, CNRS, UMR 7095, 98 bis bd Arago, 75014 Paris, France \\ ${ }^{5}$ Observatoire de Genève, Université de Genève, 51 chemin de Pégase, 1290 Versoix, Switzerland
}

5 January 2021

\begin{abstract}
With the Multi Unit Spectroscopic Explorer (MUSE), it is now possible to detect spatially extended Lyman $\alpha(\operatorname{Ly} \alpha)$ emission from individual faint $\left(M_{\mathrm{UV}} \sim-18\right)$ galaxies at redshifts, $3<z<6$, tracing gas out to circum-galactic scales comparable to the dark matter halo virial radius. To explore the implications of such observations, we present a cosmological radiation hydrodynamics simulation of a single galaxy, chosen to be typical of the Ly $\alpha$-emitting galaxies detected by MUSE in deep fields. We use this simulation to study the origin and dynamics of the high-redshift circum-galactic medium (CGM). We find that the majority of the mass in the diffuse CGM is comprised of material infalling for the first time towards the halo center, but with the inner CGM also containing a comparable amount of mass that has moved past first-pericentric passage, and is in the process of settling into a rotationally supported configuration. Making the connection to Ly $\alpha$ emission, we find that the observed extended surface brightness profile is due to a combination of three components: scattering of galactic Ly $\alpha$ emission in the CGM, in-situ emission of CGM gas (mostly infalling), and Ly $\alpha$ emission from small satellite galaxies. The weight of these contributions vary with distance from the galaxy such that (1) scattering dominates the inner regions $(r<7 \mathrm{kpc})$, at surface brightness larger than a few $10^{-19} \mathrm{cgs}$, (2) all components contribute equally around $r \sim 10 \mathrm{kpc}$ (or $\mathrm{SB} \sim 10^{-19}$ ), and (3) the contribution of small satellite galaxies takes over at large distances (or $\mathrm{SB} \sim 10^{-20}$ ). Compared to stacked MUSE observations, we show that we can reproduce the observed Ly $\alpha$ surface brightness profile closely, to within at least 0.2 dex, spanning over two orders of magnitude in surface brightness, and out to beyond the halo virial radius. We find that the CGM produces on average slightly blue-shifted (and single peaked) Ly $\alpha$ spectra, reflecting the net inflow of dense circum-galactic gas. Our simulation fails to reproduce the characteristic observed Ly $\alpha$ spectral morphology that is red-shifted with respect to the systemic velocity, with the implication that the simulation is missing an important component of neutral outflowing gas.
\end{abstract}

Key words: galaxies: formation - galaxies: evolution

\section{INTRODUCTION}

Spatially extended "haloes" of Ly $\alpha$ emission are observed around galaxies across a range of scales, ranging from massive radio

\footnotetext{
* E-mail: mitchell@strw.leidenuniv.nl
}

galaxies and quasars (e.g. Heckman et al. 1991, Reuland et al. 2003. Cantalupo et al.2014), to UV bright Lyman Break Galaxies (LBGs Steidel et al. 2011), down to comparatively UV-faint Ly $\alpha$ emitters (LAEs) (e.g. Rauch et al.2008; Matsuda et al.2012, Hayes et al. 2013, Wisotzki et al. 2016). These observations demonstrate that there must be substantial amounts of dense hydrogen gas in the circum-galactic medium (CGM) around high-redshift galaxies. Comparisons to the incidence rates of strong hydrogen absorbers 
(specifically Lyman limit systems) seen in quasar spectra support the idea that this extended emission is tracing similar gas (Wisotzki et al.2018), and direct evidence for connection between faint highredshift LAEs and strong hydrogen absorbers is now starting to appear (Lofthouse et al. 2019, Mackenzie et al. 2019, Díaz et al. 2020. Muzahid et al. 2020).

The detection of spatially extended $\operatorname{Ly} \alpha$ emission around high-redshift galaxies poses a number of questions. First, what component(s) of the CGM is being traced? Cosmological simulations predict for example the presence of inflowing filamentary streams of dense gas (e.g. Kereš et al. 2005; Dekel \& Birnboim 2006), and in addition dense gas could be either entrained or formed in situ within galactic-scale outflows (e.g. Wang 1995. Heckman et al. 2000, Scannapieco 2017). Second, a related but distinct question is what is the production mechanism for the extended Ly $\alpha$ emission? An obvious mechanism is that $\operatorname{Ly} \alpha$ is emitted within ionized HII regions inside the interstellar medium (ISM), which then resonantly scatter from neutral hydrogen in the CGM (e.g. Laursen \& Sommer-Larsen 2007; Steidel et al. 2010, Zheng et al. 2011). Alternatively, the observed photons could be emitted within the HII regions of satellite galaxies, or in situ within the CGM due to photoionization by escaping ionizing photons (fluorescence) originating from the host galaxy or quasar (e.g. Haiman \& Rees 2001, MasRibas \& Dijkstra 2016, Gallego et al. 2018), from satellites (e.g. Mas-Ribas \& Dijkstra 2016, Mas-Ribas et al. 2017), or from the wider ultraviolet background (UVB, e.g. Furlanetto et al.|2005), or because of collisional excitations and recombinations that dissipate the energy gained by compressive heating, which can be triggered by cosmological gas infall (dubbed "cooling radiation", e.g. Fardal et al. 2001, Dijkstra et al.|2006, Faucher-Giguère et al. 2010, Rosdahl \& Blaizot 2012), or by supernova shock waves that break out of the ISM (Mori et al.2004).

Steidel et al. (2010) propose that spatially extended cool gas within galactic outflows can explain simultaneously the kinematics of metal absorption lines detected "down the barrel" in the spectra of high-redshift LBGs, the Ly $\alpha$ spectra of LBGs (which are red-shifted from systemic), the equivalent width of absorbing cool gas as a function of transverse distance (detected via background sources), and Steidel et al. (2011) argue that this picture can also explain the spatially extended Ly $\alpha$ emission from the CGM (see also Dijkstra \& Kramer 2012). The question of extended Ly $\alpha$ emission aside, simple outflowing neutral gas shell models are generally used to explain the spectral morphology of observed Ly $\alpha$ spectra (e.g. Ahn 2004; Verhamme et al. 2006). In this scenario, Ly $\alpha$ photons escaping a central source backscatter from side to side of the inner surface of a circum-galactic outflowing shell, until they shift in frequency sufficiently to be out of resonance with the blueshifted side of the shell moving towards the observer. Outflowing shell models can explain the asymmetry and often complex spectral morphology of observed Ly $\alpha$ lines, the commonly observed single peaked Ly $\alpha$ line that is red-shifted from the systemic velocity, and why there is often less (or no) emission observed at (and blueward of) the systemic velocity (e.g. Verhamme et al.2008; Wofford et al. 2013. Hashimoto et al. 2015, Gronke 2017). Similar models with a more continuous expanding medium can also explain observed surface brightness profiles (e.g. Song et al. 2020).

These observations do not rule out the other scenarios however. The spectral morphology of observed Ly $\alpha$ lines could be imprinted on small scales within the ISM, rather than on CGM scales. Similarly, the spatial location of strongly outflowing gas seen down-the-barrel in galaxy spectra is highly uncertain, and could be tracing be sub-ISM scales of order $\sim 100$ pc (Chisholm et al.2016), and so not connected to the absorbing gas seen in transverse sight-lines (where the kinematics relative to the galaxy are poorly constrained). As such, the door remains open for other CGM components to provide the main explanation for the observed spatial distribution of Ly $\alpha$ haloes, without the CGM necessarily being responsible for the observed Ly $\alpha$ spectral morphology.

A natural way to explore these questions is with cosmological simulations, which in principle can simultaneously capture the complex mix of circum-galactic gas flows that surround galaxies, sourced by the filamentary infall of matter from larger scales. It must be acknowledged that the finite resolution of simulations (particularly in the CGM, see Hummels et al. 2019. Peeples et al.2019, Suresh et al. 2019, van de Voort et al. 2019) limits their ability to accurately represent multi-phase gaseous media; the resolution required to resolve the formation of dense clumps in the CGM or in winds has been estimated to be as small as $0.1 \mathrm{pc}$ (e.g. Fujita et al.2009. McCourt et al. 2018). Nonetheless, many studies of Ly $\alpha$ emission in connection to high-redshift galaxies have been performed with cosmological simulations (e.g. Cantalupo et al. 2005, Tasitsiomi 2006, Laursen \& Sommer-Larsen 2007; Zheng et al. 2010, Barnes et al. 2011), with the simulations progressively increasing in both complexity and resolution. Simulations have been used to explore the effect of scattering of centrally emitted Ly $\alpha$ photons from the CGM to create extended emission (e.g. Barnes et al. 2011, Zheng et al. 2011, Lake et al. 2015), to study in-situ gravitational "cooling" radiation (e.g. Faucher-Giguère et al.|2010, Goerdt et al. 2010, Rosdahl \& Blaizot 2012), as well as fluorescent emission powered by the ultraviolet background (UVB), and by proximate quasars (e.g. Cantalupo et al. 2005, Kollmeier et al. 2010).

Verhamme et al. (2012) use non-cosmological high-resolution simulations of idealised galaxies and emphasise the importance of allowing a cold ISM phase to form for studying Ly $\alpha$ escape from the ISM. Studies of Ly $\alpha$ transfer in cosmological simulations of high-redshift galaxies with the resolution to start addressing this challenge are now starting to appear. Behrens et al. (2019) study Ly $\alpha$ escape from a $z \sim 8$ galaxy with maximum spatial resolution of $25 \mathrm{pc}$ and gas mass resolution of $2 \times 10^{4} \mathrm{M}_{\odot}$, employing full Ly $\alpha$ radiative transfer within the ISM and CGM, but using simplified modelling to account for photo-ionization by local sources. They find generally low Ly $\alpha$ escape fractions as a result of strong local dust attenuation around young stellar clusters. Notably, Smith et al. (2019) analyse a galaxy at $z=5-7$ from the FIRE-2 suite of cosmological zoom-in simulations (Hopkins et al.2018), with a gas mass resolution of $7 \times 10^{3} \mathrm{M} \odot$. They account for photo-ionization from local sources by post-processing their simulation with ionizing UV photons, and also perform full Ly $\alpha$ radiative transfer through both the ISM and CGM. They study the angular and temporal variations of escaping $\operatorname{Ly} \alpha$ emission, finding that temporal variations are primarily driven simply by the star formation history of the primary galaxy. They also explore in post-processing the possible impact of Ly $\alpha$ radiation pressure in and around the simulated galaxy, finding that it should be dynamically important.

Here, we present results from a full cosmological radiation hydrodynamics (RHD) simulation of a high-redshift galaxy for $z \geqslant 3$, taken from the parent sample of (non-RHD) simulations presented in Mitchell et al. (2018). The simulation self-consistently includes the full range of expected Ly $\alpha$ powering mechanisms, including photo-ionization and photo-heating from local sources and the UVB. The simulation achieves a maximum spatial resolution of $14 \mathrm{pc}$ within the ISM at $z=3$, with a characteristic gas cell (and star particle) mass of $\sim 10^{3} \mathrm{M}_{\odot}$. This simulation represents a pow- 
erful tool to study the radiative transfer problem for Ly $\alpha$ photons in both the ISM and the CGM simultaneously. Our simulated galaxy (with a halo mass of $M_{200}=10^{11.1} \mathrm{M}_{\odot}$ at $z=3$ ) is deliberately chosen to be representative of the UV-faint LAEs detected by the Multi Unit Spectroscopic Explorer (MUSE) in deep MUSE fields $(\geqslant 10$ hour exposure) for $3<z<6$ (e.g. Wisotzki et al. 2016. Hashimoto et al. 2017, Inami et al. 2017, Leclercq et al. 2017). The substantial increase in sensitivity afforded by MUSE is significant in this sense, as this greatly facilitates the computational challenge of producing observable galaxies in cosmological simulations with high resolution and full radiation hydrodynamics.

Building on our previous work presented in Mitchell et al. (2018), we set out in this study to first understand what shapes the dense phases of hydrogen in the high-redshift CGM. We then use this information to interpret predictions from our simulation for spatially extended Ly $\alpha$ emission, and for the spectral morphology of the $\operatorname{Ly} \alpha$ line. We address the questions of both how Ly $\alpha$ traces the CGM, and of the origin of the extended emission (in terms of in-situ versus scattered emission that originates from the host galaxy or from satellites), albeit we do not undertake here the non-trivial task of separating the Ly $\alpha$ emitted in situ from the CGM between fluorescence and non-radiative heating sources. Complementing our study of the CGM, a forthcoming paper (Blaizot et al., in preparation) will extend the sample of radiation hydrodynamics simulations, and will focus on the observability of Ly $\alpha$ emission from the host galaxy, the angular and temporal dependence of the Ly $\alpha$ escape fraction, equivalent width, and spectral morphology, and the relative role of collisional excitations and recombinations to the escaping Ly $\alpha$ signal.

The layout of this article is as follows: we describe our simulation setup, subgrid models, and post-processing strategy in Section 2. we present our results in Section 3, we discuss various caveats and implications of these results in Section 4 , and summarise in Section 5

\section{METHODS}

\subsection{Simulation setup}

The results presented in this paper are taken from a single cosmological zoom-in simulation of a high-redshift galaxy, simulated down to $z=3$ using the RAMSES-RT code (Rosdahl et al. 2013, Rosdahl \& Teyssier 2015), which is a radiation hydrodynamics extension of the RAMSES code (Teyssier 2002). RAMSES is an Eulerian code for hydrodynamics that employs adaptive mesh refinement. The zoom-in simulation presented uses the same subgrid physics models and simulation parameters as the fiducial SPHINX simulations presented in Rosdahl et al. (2018), and effectively extends the project from $z=6$ (the final redshift currently achieved in SPHINX) to $z=3$. The initial conditions for the simulation are taken from the parent sample described in Mitchell et al. (2018), with this simulation representing a resimulation of the most massive halo from that study, with a halo mass of $M_{200}=10^{11.1} \mathrm{M}_{\odot}$ and virial radius of $R_{\mathrm{vir}}=37 \mathrm{kpc}$ at $z=3$.

We assume an underlying $\Lambda$ Cold Dark Matter cosmological model, with $\Omega_{\mathrm{M}}=0.3175, \Omega_{\Lambda}=0.6825, \Omega_{\mathrm{B}}=0.049$, $H_{0}=67.11 \mathrm{kms}^{-1} \mathrm{Mpc}^{-1}, n_{\mathrm{s}}=0.962$, and $\sigma_{8}=0.83$ (Planck Collaboration, 2014). We use the MUSIC code to generate initial conditions (Hahn \& Abel 2011). The zoom-in simulation is performed within a low-resolution box of volume $(20 \mathrm{cMpc} / h)^{3}$, with the high-resolution region selected as a sphere of $150 \mathrm{pkpc}$ radius around the target halo at $z=3$. We assume hydrogen and helium mass fractions of $X=0.76$ and $Y=0.24$ respectively, and we initialise cells with a starting metallicity of $3.2 \times 10^{-4} Z_{\odot}$, assuming the Solar metal mass fraction $Z_{\odot}=0.02$. This higher-than-pristine metallicity value is chosen to account for the enrichment from stars forming in unresolved haloes with masses below the atomic cooling limit.

For the hydrodynamics, we solve the Euler equations using a second order Gudunov scheme, using the Harten-Lax-van LeerContact (HLLC) Riemann solver with a MinMod slope limiter to construct fluid variables at cell interfaces. We employ a Courant factor of 0.8 to regulate the simulation timestep, and we assume an adiabatic index of $\gamma=5 / 3$ to close the relationship between gas pressure and internal energy, appropriate for an ideal monoatomic gas. Gravitational dynamics are solved using a particle-mesh solver and cloud-in-cell interpolation (Guillet \& Teyssier 2011). Advection of radiation between cells is solved using a first-order moment method, using the M1 closure method (Levermore 1984) and the Global-Lax-Friedrich flux function to construct the radiation field at cell interfaces.

\subsubsection{Resolution and refinement strategy}

The simulation uses a dark matter particle mass of $m_{\mathrm{DM}}=$ $10^{4} \mathrm{M}_{\odot}$ within the zoom-in region. The base refinement level of the mesh in the zoom-in region is 12 (proper cell width of $1.8 \mathrm{pkpc}$ at $z=3$ ), and it is allowed to refine up to a maximum refinement level of 19 by $z=3$, corresponding to a minimum proper cell size of 14 pkpc at $z=3$. At $z=3$ there are $1.2 \times 10^{7}$ leaf cells within $R_{\text {vir }}$, the median cell width in the diffuse CGM $\left(0.2<r / R_{\text {vir }}<\right.$ 1 , excluding satellite galaxies) is $227 \mathrm{pc}$ (weighted by mass) and $454 \mathrm{pc}$ (weighted by volume), and the median cell size in the ISM $\left(r<0.2 R_{\text {vir }}\right.$ ) is $14 \mathrm{pc}$ (weighted by mass) and $113 \mathrm{pc}$ (weighted by volume).

Cells are refined either if: i) $M_{\mathrm{DM}, \text { cell }}+\frac{\Omega_{\mathrm{M}}}{\Omega_{\mathrm{B}}} M_{\text {baryon,cell }}>$ $8 m_{\mathrm{DM}}$, where $M_{\mathrm{DM} \text {, cell }}$ and $M_{\text {baryon,cell }}$ are respectively the total dark matter and baryonic mass enclosed within a cell, ii) the cell width is larger than one quarter of the Jeans length $\lambda_{\mathrm{J}} \equiv \sqrt{\frac{\pi c_{\mathrm{s}}^{2}}{G \rho_{\text {gas }}}}$, where $c_{\mathrm{s}}$ is the gas sound speed, $\rho_{\text {gas }}$ is the gas mass density, and $G$ is the gravitational constant. We do not impose an equation of state to artificially pressurize the ISM if the Jeans length is unresolved, which can occur when the Jeans length is smaller than the minimum allowed cell size.

\subsubsection{Radiation and thermochemistry}

To represent the locally produced ultraviolet (UV) radiation field, we employ three discrete radiation bins, with frequencies bracketed by the ionization energies of hydrogen and helium, including both the first and second ionization state. Full non-equilibrium interactions between ionizing UV photons, hydrogen and helium are modelled on the fly as described in Rosdahl et al. (2013); Rosdahl \& Teyssier (2015), including the effects of photo-ionization, heating and momentum transfer. The gas temperature and ionization states of hydrogen and helium are all tracked and updated accordingly. Radiation advection is subcycled relative to the hydrodynamical timestep, and we use a reduced speed of light approximation with an effective advection velocity of 1/80 times the true speed of light. As described in Rosdahl et al. (2018), we use BPASs stellar evolution models (Eldridge et al. 2008) to set the input spectral energy distribution of star particles. We use BPASS version 2.0 (Eldridge 
\& Stanway 2016, Stanway et al. 2016) which assumes a $100 \%$ binary fraction. Since our zoom-in simulation targets only a single galaxy, we include the effects of a homogeneous evolving ultraviolet background (UVB) radiation field, in addition to the radiation provided by local stellar sources. We adopt the model for the UVB from Faucher-Giguère et al. (2009), but apply a self-shielding correction that exponentially damps the UVB intensity for gas cells with $n_{\mathrm{H}}>10^{-2} \mathrm{~cm}^{-3}$ (Faucher-Giguère et al. 2010, Rosdahl \& Blaizot 2012).

In addition to the full non-equilibrium thermochemistry solved for hydrogen and helium, metal line cooling is modelled for gas with temperature $T>10^{4} \mathrm{~K}$ using CLOUDY (Ferland et al. 1998), assuming ionization equilibrium with a Haardt \& Madau (1996) UVB (and so is not modelled self-consistently with the non-equilibrium hydrogen and helium thermochemistry). For $T<10^{4} \mathrm{~K}$ we apply fine structure cooling rates from Rosen \& Bregman (1995).

\subsubsection{Star formation}

We use a model for star formation inspired by Federrath \& Klessen (2012), as described in Kimm et al. (2017), Trebitsch et al. (2017), and Rosdahl et al.(2018). The model accounts for the stabilising effects of both thermal pressure and turbulent motions against gravitational collapse. The precise criteria for stars to form in a cell are that: $(i)$, the local hydrogen number density $n_{\mathrm{H}}>10 \mathrm{~cm}^{-3}$ (and that the local overdensity is greater than 200 times the cosmic mean), (ii), the gas is locally convergent and the host cell represents a local density maxima compared to the six nearest cells, (iii), the cell width $\Delta x$ is smaller than the turbulent Jeans length given by

$$
\lambda_{\mathrm{J}, \mathrm{turb}}=\frac{\pi \sigma_{\mathrm{gas}}^{2}+\sqrt{36 \pi c_{\mathrm{s}}^{2} G \Delta x^{2} \rho+\pi^{2} \sigma_{\mathrm{gas}}^{4}}}{6 G \rho \Delta x},
$$

where $\sigma_{\text {gas }}$ is the local gas velocity dispersion, computed using the velocity gradients to cells that share vertices with the host cell. As described in Rosdahl et al. (2018), the computation of $\sigma_{\text {gas }}$ is modified with respect to the model described in Kimm et al. (2017) by subtracting rotational and symmetric divergent components of the velocity field, such that the turbulent support against a converging flow is only provided by the relevant anisotropic motions.

For cells that meet these criteria, star particles are allowed to form stochastically with (on average) a rate given by $\dot{\rho}_{\star}=$ $\epsilon_{\star} \rho_{\text {gas }} / t_{\text {ff }}$, where $t_{\text {ff }}=\left[3 \pi /\left(32 G \rho_{\text {gas }}\right)\right]^{1 / 2}$ is the local freefall time, and $\epsilon_{\star}$ is a variable efficiency that depends on the local turbulent properties of the gas and on the sound speed (see Kimm et al. 2017, Trebitsch et al. 2017, for details). This efficiency can often reach or exceed values of unity, leading to a bursty scenario for star formation.

\subsubsection{Stellar evolution and supernova feedback}

We model the effects of supernova explosions using the mechanical feedback scheme described in Kimm \& Cen (2014) and Kimm et al. (2015); specifically we use the mechanical feedback scheme with multiple explosions from the list of variant models considered in Kimm et al. (2015). For each star particle with age $<50 \mathrm{Myr}$ and $>3 \mathrm{Myr}$, a stochastic sampling is performed over the supernova delay time distribution, with individual supernova explosions of $10^{51} \mathrm{erg}$ that inject energy/momentum into the host cell and neighbouring cells around the particle. We assume a Kroupa(2002) stellar initial mass function, with $20 \%$ of the star particle mass returned to the neighbouring cells across all supernova events, and in addition metals are injected with a metal yield $y=0.075$. As discussed in Rosdahl et al. (2018) and Mitchell et al. (2018), we break consistency with the Kroupa stellar initial mass function (IMF) to decide how many supernova explosions occur for each star particle, increasing the number by a factor four such that there are four supernova explosions (each of $10^{51} \mathrm{erg}$ ) per 100 Solar masses of stars formed. This choice was made by Rosdahl et al. (2018) to calibrate the SPHINX simulations against observational constraints for $z \geqslant 6$ and was made by Mitchell et al. (2018) to improve agreement with constraints for $z \geqslant 3$.

Depending on the cell metallicities and densities, the mechanical scheme determines for each cell whether the simulation is able to resolve the adiabatic phase (in which case the injected energy is $10^{51} \mathrm{erg}$ and the injected momentum scales $\propto \sqrt{\mathrm{m}}$, where $m$ is the mass of the neighbouring cells that is considered to be entrained into the outflow), and otherwise injects energy and the maximum momentum achieved in the momentum-conserving snowplow phase, with a maximum injected momentum of $3 \times 10^{5} \mathrm{M}_{\odot} \mathrm{kms}^{-1}$ for gas with $n_{\mathrm{H}}=1 \mathrm{~cm}^{-3}$ and $Z=Z_{\odot}$ (Blondin et al. 1998, Thornton et al. 1998). Following Kimm et al. (2017), we then boost the maximum momentum to $4.2 \times 10^{5} \mathrm{M}_{\odot} \mathrm{kms}^{-1}$ if the local Stromgren radius is unresolved, accounting for unresolved photoionizing heating from the star particle, which can reduce the densities around the star and so reduce the radiative losses (Geen et al. 2015).

\subsection{Halo finder and satellite identification}

We identify both central and satellite dark matter subhaloes using the AdaptaHOP algorithm (Aubert et al. 2004), and construct merger trees following Tweed et al. (2009). We store simulation outputs with a temporal cadence of $10 \mathrm{Myr}$. Halo centres are defined based on the dark matter density maxima. All halo masses $\left(M_{200}\right)$ and virial radii $\left(R_{\text {vir }}\right)$ quoted are measured by computing the spherical radius within which the mean enclosed density is 200 times the critical density of the Universe.

For each satellite identified by the halo finder, we assign particles and gas cells to that satellite if they fall within the tidal radius, which we approximate following Binney \& Tremaine (2008) as

$$
r_{\mathrm{t}}=R_{0}\left(\frac{m}{M\left(R_{0}\right)\left(3-\left.\frac{\mathrm{d} \ln M}{\mathrm{~d} \ln (R)}\right|_{R=R_{0}}\right)}\right)^{1 / 3},
$$

where $R_{0}$ is the distance from the satellite centre to the host centre, $M\left(R_{0}\right)$ is the mass of the host enclosed within $R_{0}$ and $m$ is the mass of the satellite (the mass within $r_{\mathrm{t}}$ ). This relation is appropriate for a satellite on a circular orbit within a spherically symmetric host potential.

\subsection{Tracer particles and classifying circum-galactic gas}

The results presented in this paper make extensive use of tracer particles to provide Lagrangian information about the trajectories of gas in our simulation. We use the Monte Carlo implementation of tracer particles introduced by Genel et al. (2013), and adapted for use in RAMSES simulations by Cadiou et al. (2019). Cadiou et al. (2019) show that these tracers accurately reproduce the underlying gas density field, and self-consistently follow our subgrid models 
for star formation and feedback. We use a relatively high tracer sampling, with ten tracers initialised per gas cell in the zoom-in region of the initial conditions.

One application of tracer particles is to classify the nature of the gas contained within a given cell in the simulation. We can then use these components to (for example) decompose Ly $\alpha$ emission from the CGM into different components, thereby gaining insight into the CGM-Ly $\alpha$ connection. Unless otherwise stated, we use the following definitions to define different (mutually exclusive) gaseous components within the virial radius of the primary host halo:

- ISM: any gas with radius $r<0.2 R_{\mathrm{vir}}$. In practice this also includes material that could be argued to belong to an ISM-CGM interface, or to the inner CGM, but this is qualitatively unimportant for our results.

- Satellite gas: any gas that is within the tidal radius of a satellite galaxy.

- Stripped CGM: circum-galactic gas that was within the tidal radius of a satellite (for at least $30 \mathrm{Myr}$ ) as it entered the virial radius of the host halo, but is subsequently located beyond the tidal radius. This could be related to ram pressure stripping, gravitational tidal forces, or stellar feedback from the satellite; we do not attempt to distinguish between these mechanisms.

- First-infall CGM: circum-galactic gas that is radially infalling from the inter-galactic medium (IGM) for the first time. We account for occasional fluctuations in the radial velocity of tracers by requiring that a tracer be radially outflowing consecutively for $30 \mathrm{Myr}$ after the current snapshot in order to have left the "firstinfall" component at the current snapshot.

- Post-pericentric CGM: circum-galactic gas that has moved past pericenter of the orbit associated with first infall. This material can be outflowing (after pericenter) or inflowing (after apocenter). Diffuse gas that has been inside the CGM for more than a halo dynamical time will almost always belong to this component, unless it is identified as having been influenced by feedback, or was stripped from a satellite.

- Feedback-influenced CGM: circum-galactic gas that has been positively identified as having been likely influenced by stellar feedback in the past. This category overrides all of the other CGM classifications.

Gas outside the virial radius of the primary host halo is always labelled as "first-infall", unless it is within the virial radius of another halo (in which case it is then labelled "satellite gas"), or was ejected from the primary host. Note therefore that when considering $\operatorname{Ly} \alpha$ surface brightness profiles that extend beyond the virial radius, we label Ly $\alpha$ photons as connected to "satellites" if they are either associated to a satellite subhalo of the primary, or if they are associated with a neighbouring halo outside of the virial radius of the primary halo.

We estimate which tracers have been influenced by feedback (within the main halo) at a given snapshot by requiring that all of the following conditions are met:

- the gas is outflowing with a radial velocity (relative to the primary host halo center) of $v_{\mathrm{r}}>50 \mathrm{kms}^{-1}$,

- the gas has accelerated radially by $\Delta v_{\mathrm{r}}>30 \mathrm{kms}^{-1}$ over the last $10 \mathrm{Myr}$, and the gas has moved outwards by $\Delta r>0.1 R_{\mathrm{vir}}$ over the same interval,

- the radial kinetic plus thermal specific energy of the gas has increased by at least $25 \%$ over the same interval,

- the tracer is not going to enter within the tidal radius of a satel- lite over the next 30 Myr. This can be important because the halo finder often loses track of satellites within the inner regions of the host halo,

- the tracer has not accelerated radially outwards simply due to moving past pericenter of its (gravity-driven) orbit.

We estimate if tracers are undergoing such an orbital transition at a given snapshot by first computing the minimum radial velocity (where inflowing is negative) of the tracer at previous snapshots, searching back in time for as long as the tracer velocity is monotonically decreasing. For a particle that has just moved past pericenter, this procedure identifies the maximum past infall velocity (and will return a smaller absolute value for particles that fluctuate in velocity while settled within the ISM, corresponding roughly to the ISM velocity dispersion). We then search for the corresponding maximum radial velocity of the tracer for future snapshots, searching forwards for as long as the radial velocity is monotonically increasing with time. This then returns the maximum future outflowing velocity of the particle before it starts to slow as it approaches apocenter. For particles that are on pure infalling orbits (independent of feedback or other energy injection mechanisms), the maximum future outflow velocity will always be similar or less than the maximum past inflow velocity, and so for the tracer to be considered influenced by feedback we require that the maximum future outflow velocity be greater than $3 / 2$ times the magnitude of the maximum past infall velocity.

This scheme is of course approximate, and was designed and tested primarily by manual inspection of many individual tracer trajectories. In practice, feedback events will affect many more of the tracers in the simulation at some level, and there can be other physical mechanisms that might cause a tracer to suddenly accelerate outwards.

\subsection{Ly $\alpha$ emission and radiative transfer}

With a full radiation hydrodynamics simulation, we selfconsistently account for both the photo-ionization and photoheating of hydrogen by local stellar source ${ }^{1}$ (plus a uniform UVB), and so we can compute the Ly $\alpha$ luminosity of all gas cells within the zoom-in region based on the relevant collisional excitation and recombination rates.

For recombinations, we assume case B (see Blaizot et al., in prep for a full justification of this choice) and follow Cantalupo et al. (2008), computing the rate of Ly $\alpha$ photons produced by recombinations from a single gas cell as

$$
\varepsilon_{\mathrm{rec}}=n_{\mathrm{e}} n_{\mathrm{p}} \epsilon_{\mathrm{Ly} \alpha}^{B}(T) \alpha_{B}(T) \times(\Delta x)^{3},
$$

where $n_{\mathrm{e}}$ and $n_{\mathrm{p}}$ are the electron and proton number densities, $\alpha_{B}(T)$ is the case $\mathrm{B}$ recombination rate, $\epsilon_{\mathrm{Ly} \alpha}^{B}(T)$ is the fraction of recombinations that result in the production of a $\operatorname{Ly} \alpha$ photon, and $(\Delta x)^{3}$ is the volume of the cell. We evaluate $\epsilon_{\mathrm{Ly} \alpha}^{B}(T)$ using the fit from (Cantalupo et al. 2008 given by their equation 2), and $\alpha_{B}(T)$ with the fit from Hui \& Gnedin (1997, their appendix A).

For collisional excitations, we compute the rate of resulting Ly $\alpha$ photons produced from a single cell as

1 Note that we do not model supermassive black holes in our simulation, and so we do not account for photo-heating and ionization by any local active galactic nuclei. 


$$
\varepsilon_{\mathrm{col}}=n_{\mathrm{e}} n_{\mathrm{HI}} C_{\mathrm{Ly} \alpha}(T) \times(\Delta x)^{3},
$$

where $n_{\mathrm{HI}}$ is the number density of neutral hydrogen atoms, and $C_{\mathrm{Ly} \alpha}(T)$ is the rate of collisional excitations from level $1 s$ to $2 p$, which we evaluate using the fit from Goerdt et al. (2010 their equation 10). In addition, we set the collisional excitation rate to zero for cells within which the net cooling timescale is less than ten times the simulation timestep, defining the net cooling timescale as $t_{\text {cool, net }} \equiv(\mathcal{C}-\mathcal{H})^{-1}$, where $\mathcal{C}$ and $\mathcal{H}$ are the cooling and heating rates. In such cells, we do not adequately resolve the balance between heating and cooling, which may lead to a significant overestimate or underestimate of the true collisional excitation rate. Setting the collisional excitation rate to zero means that we are computing a conservative lower limit for these cells. We do however resolve the net cooling timescale in at least $90 \%$ of the cells within the virial radius, and so we do not believe that this introduces a large uncertainty to the collisional excitation contribution to Ly $\alpha$ (Blaizot et al., in prep).

With the Ly $\alpha$ emission rates from recombinations and collisional excitations, we then perform Monte Carlo radiative transfer in post-processing to compute the Ly $\alpha$ signal that escapes the halo, using the RASCAS code (Michel-Dansac et al. 2020). We sample each emission component $\left(\varepsilon_{\text {rec }}\right.$ and $\left.\varepsilon_{\text {col }}\right)$ with $4 \times 10^{5}$ Monte Carlo photon packets cast from cells with a probability proportional to their luminosity. Each photon packet is emitted with a frequency in the frame of the emitting cell which is drawn from a Gaussian distribution of width fixed by the cell's temperature (see Sec. 2.2 of Michel-Dansac et al. 2020). In order to measure the extended emission out to large distances, all cells within a radius of 11 arcsec around the main galaxy are allowed to emit. In turn, photon packets are propagated until they either pass that radius or are absorbed by a dust grain. We have included dust following the model of Laursen et al. (2009b), assuming SMC dust properties. In this model, the amount of dust scales primarily with the amount of neutral hydrogen and of metals, although traces of dust will be present in the ionised phase as well. We refer to Blaizot et al. (in prep.) for a detailed discussion of the effect of dust on the Ly $\alpha$ properties of our simulated galaxy. This model results in UV attenuations (typically $\sim 1-2$ mag at $M_{1500} \sim-20 \mathrm{AB}$ ) and Ly $\alpha$ escape fractions (typically a few percent) which are broadly consistent with what is observed for LAEs. In the scope of the present paper, the main effect of dust is to limit the Ly $\alpha$ flux emerging from the ISM of the central plus satellite galaxies.

In order to accelerate the computation, we use the coreskipping algorithm described in Smith et al. 2015. Sect. 3.2.4), which very slightly underestimates the effect of dust (introducing a relative error of order $1 \%$ on the escape fraction of $\operatorname{Ly} \alpha$ radiation) but produces a speedup of the computation of a factor $\sim 1000$. We do not apply any attenuation correction for the intergalactic medium, but this is generally expected to have at most a modest $20-30 \%$ effect for wavelengths shorter than the line center for the redshifts $(z \sim 3)$ studied here (e.g. Inoue et al. 2014; Hayes et al. 2020).

The main output of RASCAS is the state of photon packets as they escape the computational domain. With this data, we can perform part of our analysis and for example understand how different gas phases contribute to the observed extended emission. This does however limit our analysis to angle-averaged properties of the Ly $\alpha$ emission, which to some extent inhibits our ability to compare our simulation directly to MUSE observations. In Appendix A we make use of the peeling algorithm (Yusef-Zadeh et al. 1984.
Zheng \& Miralda-Escudé 2002), as described in Dijkstra (2017, Sect. 9.3), to collect flux in 12 mock MUSE data-cubes at each snapshot. These 12 mocks correspond to observations of the simulated galaxy from 12 different directions (approximately equally spaced, with a common origin, and that are the same for all redshift outputs), allowing us to more closely reproduce the observational selection and analysis of Wisotzki et al. (2018).

\section{RESULTS}

Our results are presented as follows: we start by using tracer particles to explore the origin of the dense gas phases of the CGM in Section 3.1. we connect this to the properties of Ly $\alpha$ emission related to the CGM in Section 3.2, we decompose the different components that comprise observed Ly $\alpha$ haloes in Section 3.3 we compare Ly $\alpha$ surface brightness profiles with stacked MUSE observations in Section 3.4 and we finish by presenting the predicted Ly $\alpha$ spectral morphology in Section 3.5. Since our focus is primarily directed at the connection between $\operatorname{Ly} \alpha$ emission and the circum-galactic medium, we generally exclude gas (and photons) associated with satellite galaxies for the analysis presented in Sections 3.1 and 3.2 We then introduce the contribution of satellites when considering the observed properties of our simulated galaxy, in Sections 3.3 3.4 and 3.5

\subsection{Lagrangian origin of the neutral hydrogen in the CGM}

In Mitchell et al. (2018), we analysed the properties of circumgalactic gas from a sample of zoom-in simulations at $z \approx 3$, including the halo studied here. Focussing on the neutral phase of hydrogen (which we expect to be the closely related to the gas phases associated with extended Ly $\alpha$ emission), we found that the mass per unit radius in neutral hydrogen ${ }^{2}$ drops exponentially as a function of radius. In contrast, the mass profile of warm and hot ionized CGM components is generally flat or rising with radius. This is shown for the simulation studied here in the top panel of Fig. 1 Most of the neutral hydrogen is radially inflowing (dashed blue line), but a significant fraction $(\approx 1 / 3)$ is outflowing (solid blue line) in the inner regions $\left(r<0.5 R_{\text {vir }}\right)$. Under the approximation of constant radial flow velocity, a drop in the neutral hydrogen mass with radius implies that mass is being lost from the phase as gas flows outward, and correspondingly that the inflowing neutral component is gaining mass as gas flows inwards. This is confirmed by the lower panel of Fig. 1 which shows similar gradients for the radial momentum profiles.

Understanding this behaviour is an important prerequisite for understanding in turn the predicted spatially extended Ly $\alpha$ signal. Our simulation predicts copious amounts of outflowing neutral hydrogen in the inner CGM, which may be consistent with the picture of outflowing neutral gas shells that is commonly invoked to explain the Ly $\alpha$ spectral morphology of observed LAEs (e.g. Ahn 2004, Verhamme et al. 2006). At larger radii ( $r>0.5 R_{\text {vir }}$ ), there is comparatively very little neutral hydrogen in our simulation, which poses the question of whether the outflowing neutral gas recorded in the inner CGM is being photo-ionized as it moves outwards (joining the warm-ionized component, solid green line,

2 By neutral neutral hydrogen mass we mean $0.76 x_{\mathrm{HI}} \rho_{\text {gas }} \Delta x^{3}$, where 0.76 is the assumed primordial hydrogen mass fraction, and $x_{\mathrm{HI}}$ is the hydrogen neutral fraction of a given cell. 


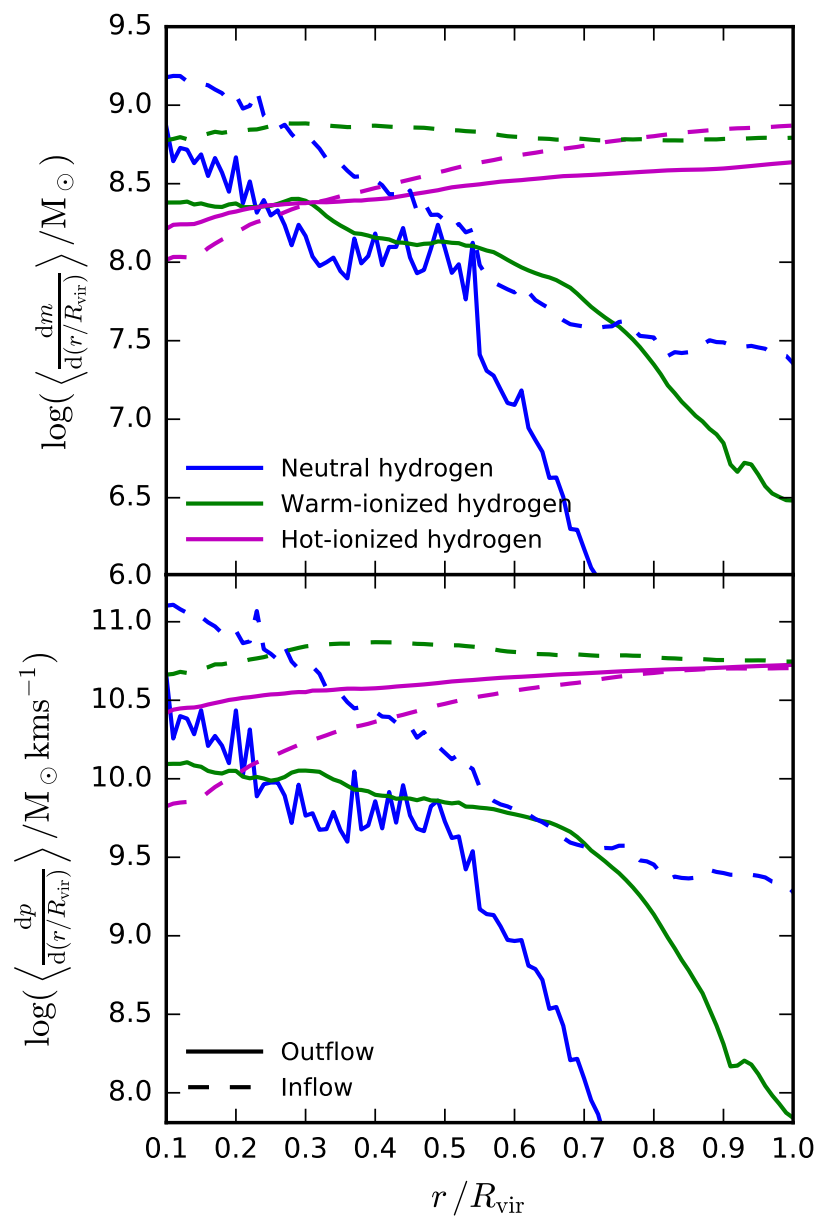

Figure 1. Mass and radial momentum profiles of circum-galactic gas (excluding satellite galaxies), plotted as a function of radius. Data is meanstacked over all available outputs between $z=4$ and $z=3$. Solid lines show outflowing gas, and dashed lines show inflowing gas. Blue shows neutral hydrogen (i.e. the mass/momentum of hydrogen in each cell multiplied by the neutral hydrogen fraction), green shows warm-ionized hydrogen $\left(T<10^{4.5} \mathrm{~K}\right)$, and magenta lines show hot-ionized hydrogen $\left(T>10^{4.5} \mathrm{~K}\right)$. Most of the neutral hydrogen content of the CGM is located within the inner halo, whereas the hot-ionized profile rises with radius, comprising the majority of circum-galactic gas in the outer halo. The sharp drop in neutral hydrogen mass/momentum with radius could be associated with a galactic fountain process, or with a change in ionization state of gas as it flows inwards/outwards.

which is more radially extended than the neutral outflowing component), or alternatively is falling back towards the ISM in a fountain flow (before reaching the outer CGM). Accordingly, the increase in inflowing neutral gas in the inner CGM could be attributed to a change in ionization state of gas that is infalling for the first time, or could be connected to the afore-mentioned fountain flow of neutral gas. Another question that arises is whether the neutral hydrogen content of the CGM in the simulation is being strongly affected by feedback. In Mitchell et al. (2018), we show that not including stellar feedback in our simulations approximately leaves the neutral content of the CGM unchanged, implying that neutral circumgalactic gas flows in the simulation are driven primarily by other processes.

We can now address these questions directly by using the Lagrangian information afforded by tracer particles. Fig. 2 shows the
Lagrangian history of gas selected as it crosses either the virial radius, or a surface at $0.3 R_{\text {vir }}$. We temporally stack simulation outputs around the crossing redshift, such that the trajectory distributions are representative for this halo over $3.5<z<4$. Focussing first on inflowing neutral hydrogen crossing $0.3 R_{\text {vir }}$ (far-left column), the distribution of tracer trajectories shows that most of the selected gas is flowing inwards for the first time from the wider environment, as opposed to being recycled in a fountain flow. The temperature of the inflowing gas ( $T \sim 10^{4} \mathrm{~K}$, middle row) is fairly steady as the gas flows inwards down to $r \sim 0.5 R_{\mathrm{vir}}$, consistent with it being maintained in thermal equilibrium by photo-heating. At the same time, the density of the gas gradually increases (fourth row) until the gas reaches the threshold to be self-shielded from ionizing radiation at $n_{\mathrm{H}} \sim 10^{-2} \mathrm{~cm}^{-3}$, at which point the neutral hydrogen fraction rapidly increases (second row). From this, we conclude that the exponential increase in the mass/momentum of inflowing neutral hydrogen with decreasing radius seen in Fig. 1 is caused primarily by compression of infalling gas leading to a change of ionization state ${ }^{3}$

Shifting our attention to the neutral outflowing hydrogen crossing $0.3 R_{\text {vir }}$ (second-left column in Fig. 2), the past distribution of trajectories shows that this gas is comprised primarily of infalling gas that has moved past first pericenter. The majority of this outflowing gas will soon move past apocentre of its first orbit and will subsequently settle down towards the central ISM. The median average ionization fraction stays at $x_{\mathrm{HI}} \sim 0.8$ after the selection redshift, meaning that the sharp drop in outflowing hydrogen mass with increasing radius is primarily connected to gas falling back down onto the ISM, rather than because of a change in ionization state. Employing the Lagrangian tracer classifications described in Section 2.3 this component of the gas is, as expected, associated with the "post-pericenter" component (green line in the bottom row), which comprises the majority of the neutral outflow at $0.3 R_{\text {vir. }}$. At the same time, a subset of the gas does move out into the outer CGM, reflected by the upper $\left(84^{\text {th }}\right)$ percentile in the radius distribution (top-row). This is associated with a growth in the "feedback-influenced" component (red line in the bottom row), implying that feedback is responsible for pushing this fraction of the gas out into the outer halo.

The third and fourth columns of Fig. 2 give a broader perspective by looking at the trajectories of gas before/after crossing the halo virial radius (in this case without selecting any specific gas phase). Starting with inflowing gas at the virial radius (third column), the future trajectories of the gas are diverse, with $\approx 40 \%$ of the gas settling into the central ISM (orange line, bottom row), $\approx 20 \%$ moving past pericenter but remaining in the inner CGM (green line, bottom row), and $\approx 20 \%$ moving back out of the halo, which is connected with the component that is estimated to have been influenced by feedback (red line, bottom row). This diversity is reflected by a broad range in temperature and density, spanning three orders of magnitude in temperature and seven orders of magnitude in density (at $300 \mathrm{Myr}$ after entering the halo). It is worth noting that the inflowing gas is almost entirely ionized at the virial radius, and on average remains mostly ionized for at least $300 \mathrm{Myr}$

\footnotetext{
3 Note however that the distributions shown in Fig. 2 are weighted by flux, which will somewhat down-weight (relative to weighting by mass) the importance of a neutral fountain flow that is turning around at a radius comparable to $0.3 R_{\text {vir }}$. Fig. 1 does indeed show a jump in both the inflowing and (more prominently) the outflowing neutral hydrogen radial mass profiles at $r \approx 0.5 R_{\mathrm{vir}}$, implying that fountain flows do indeed also play a role.
} 


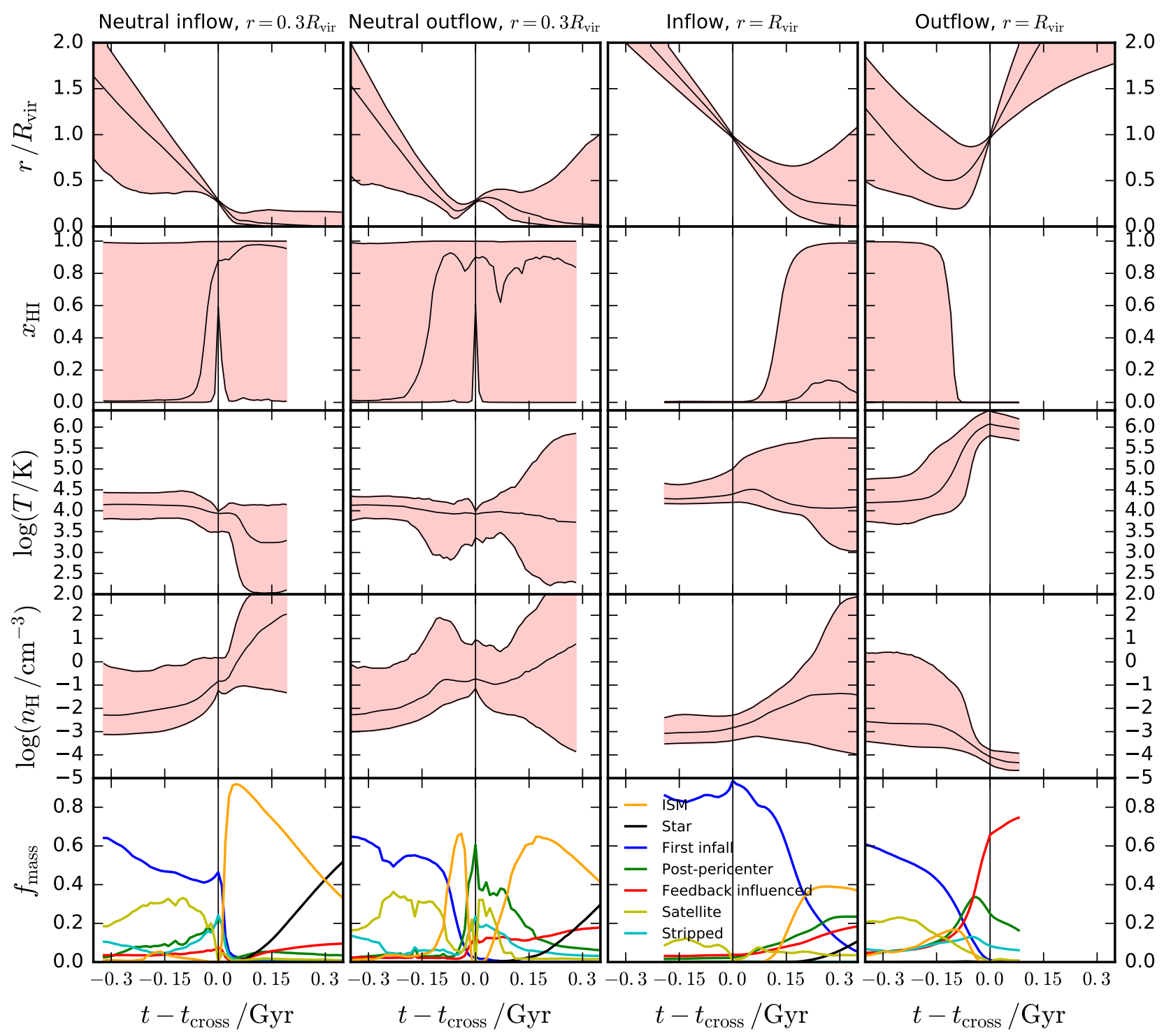

Figure 2. An overview of the Lagrangian history of circum-galactic gas in the simulation. Each panel shows weighted distributions of tracer trajectories, for tracers that meet sets of selection criteria. Each column corresponds to a different set of selection criteria, as indicated by the column titles. Selections are performed either at $0.25<r / R_{\mathrm{vir}}<0.3$, or at $0.95<r / R_{\mathrm{vir}}<1$, and include either inflowing or outflowing gas. Individual tracers that fulfil these criteria are shifted along the $\mathrm{x}$-axis such that they pass the selection at $t=t_{\text {cross. }}$. Tracer distributions are flux-weighted either by the total radial momentum at the time of selection (right-side columns), or by the radial momentum of neutral hydrogen at the time of selection (left-side columns). Solid black lines indicate the $16^{\text {th }}, 50^{\text {th }}$, and $84^{\text {th }}$ percentiles of the distributions. Each row corresponds to a different gas quantity, including radius, neutral hydrogen fraction, temperature, density, and the mass fraction, $f_{\text {mass }}$, belonging to the discrete and mutually exclusive Lagrangian components described in Section 2.3 Lagrangian components include gas that is infalling for the first time (blue), that has been stripped from a satellite (cyan), that is within the tidal radius of a satellite (cyan), that has been influenced by feedback (red), that has moved past the first pericentric passage (green), that belongs to the central ISM (orange), that belongs to a satellite (khaki), and tracers that are locked inside stars (black). Distributions of gas temperature, density, and ionization fraction are truncated once $20 \%$ of the selected gas has either been locked into stars (first, second columns), or is located beyond $2 R_{\mathrm{vir}}$ (third, fourth columns), at which we point we stop tracking trajectories.

after entering the halo (so for at least one to two halo dynamical times).

Considering instead outflowing gas at the virial radius (farright column), this selection is dominated by the feedback influenced component (red line, bottom row). The majority of this gas did not come from the ISM, but was instead accelerated outwards while infalling (for the first time) within the CGM, with an associated increase in temperature from $T \sim 10^{4} \mathrm{~K}$ to $10^{6} \mathrm{~K}$, consis- tent with being heated and entrained by hot and diffuse supernovadriven outflows. Half of this component did not even reach $r<$ $0.5 R_{\text {vir }}$ before being accelerated outwards.

\subsection{The composition of the high-redshift $\operatorname{Ly} \alpha$-emitting CGM}

Combined together, Fig. 1 and Fig. 2 present a picture in which most of the the neutral (and so higher-density) phase of the CGM 

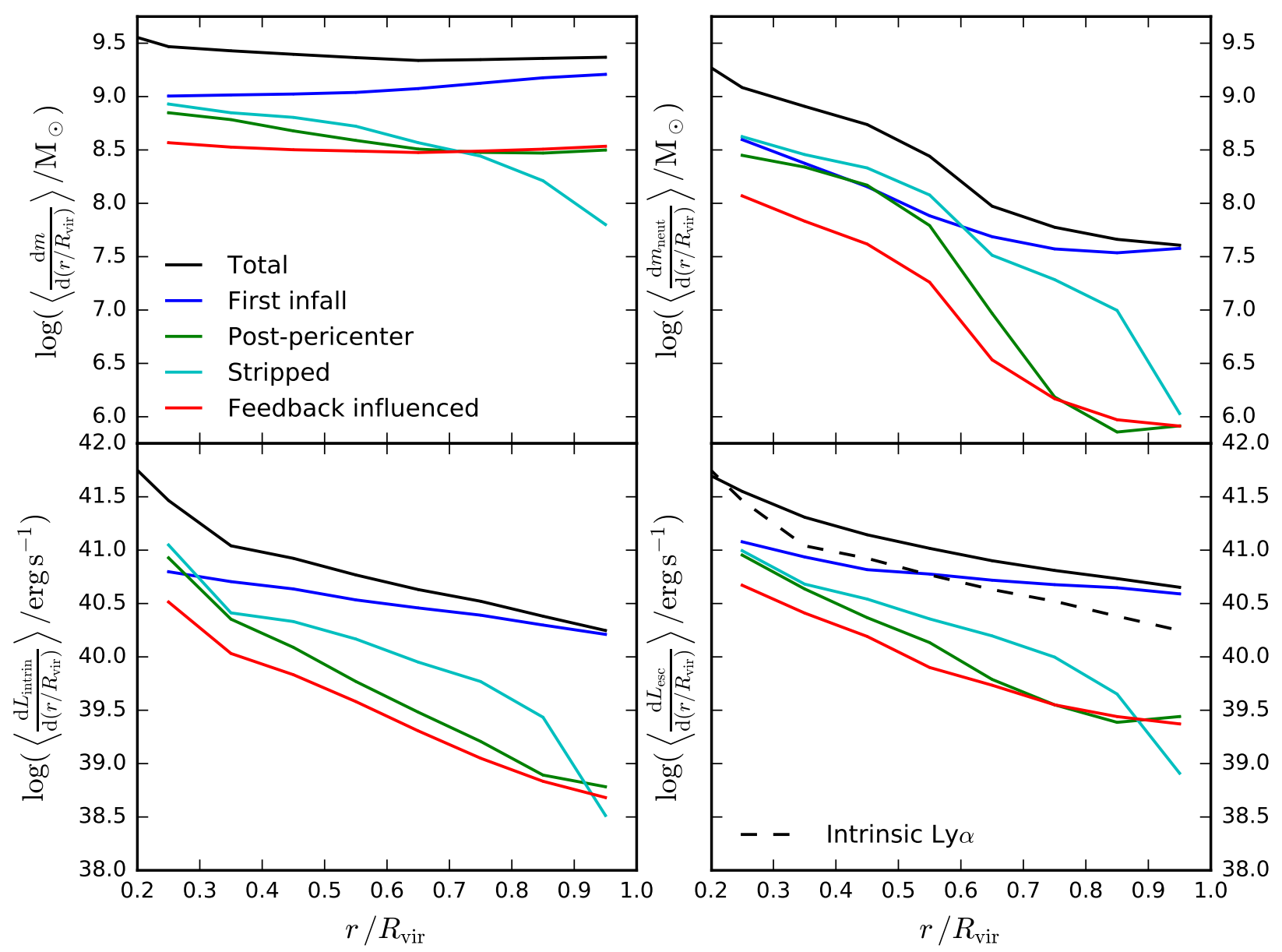

Figure 3. Radial profiles of total gas mass (upper-left), neutral hydrogen mass (upper-right), and both intrinsic (lower-left) and escaping Ly $\alpha$ luminosity (lower-right) for circum-galactic gas (excluding satellite galaxies). Unlike in Fig. 1 here the radial profiles are split between different Lagrangian CGM components, as indicated by the legend. Data is mean-stacked over all available outputs between $z=4$ and $z=3$. The intrinsic Ly $\alpha$ luminosity profile (lower-left) shows the luminosity of emitting gas, irrespective of whether photons escape the halo. The escaping Ly $\alpha$ luminosity profile (lower-right) shows how escaping Ly $\alpha$ photons last trace the CGM before escape, meaning that photons are binned at the radial position of last-scattering (or at the emission location if no scattering occurs), and are assigned to the CGM component associated with that position. The dashed black line in the lower-right panel shows the total intrinsic Ly $\alpha$ luminosity profile from the lower-left panel, for comparison. Note that these the profiles are binned as a function of three-dimensional radius, and so the Ly $\alpha$ profiles are not directly comparable to observed surface brightness profiles. Ly $\alpha$ emission is dominated by first-infalling gas in the outer CGM, both in terms of intrinsic luminosity and as the component being traced prior to escape. Ly $\alpha$ emission in the inner CGM traces gas associated with a mix of components. Radiative transfer effects (i.e. scattering) enhances the escaping Ly $\alpha$ profile relative to intrinsic by around a factor two in the CGM.

is undergoing gravitational infall, with the majority of neutral inflows comprised of gas infalling before first-pericentric passage, and the majority of neutral outflowing gas comprised of gas that has moved past first pericenter, and is in the process of settling into an equilibrium configuration either within the inner CGM, or down in the dense ISM at the halo centre.

\subsubsection{Radial profiles}

This picture is shown explicitly in Fig. 3, which splits the total CGM (top-left panel) and neutral hydrogen content of the CGM (top-right) between the various Lagrangian components described in Section 2.3 The total gas content (top-left panel) of the outer CGM is dominated by the inflowing gas that is infalling for the first time ("first infall", blue line), with the contribution from gas that has moved past first pericentric passage ("post pericenter", green line) gradually increasing until it becomes comparable to the first- infall component at $r \sim 0.2 R_{\mathrm{vir}}$. Gas that has been stripped/removed from satellites ("stripped", cyan line) is also an important contributor in the inner CGM (and in practice is comprised of both gas that is infalling for the first time, and gas that has moved its first pericentric passage). Gas that has been estimated to have been clearly influenced by stellar feedback ("feedback influenced", red line) is subdominant at all radii, but with a flat radial mass profile, corresponding to the approximately flat mass and momentum profiles seen for the hot-ionized outflowing phase in Fig. 1

Shifting attention to the neutral hydrogen content of the CGM (top-right panel in Fig. 3, the drop in neutral hydrogen mass with radius is evident for each individual Lagrangian component. The relative contribution of stripped gas is higher for the neutral phase, and there is a greater level of parity between the first infall, post pericenter, and stripped components, particularly within $r<0.6 R_{\mathrm{vir}}$. At larger radii, the first-infalling component increas- 

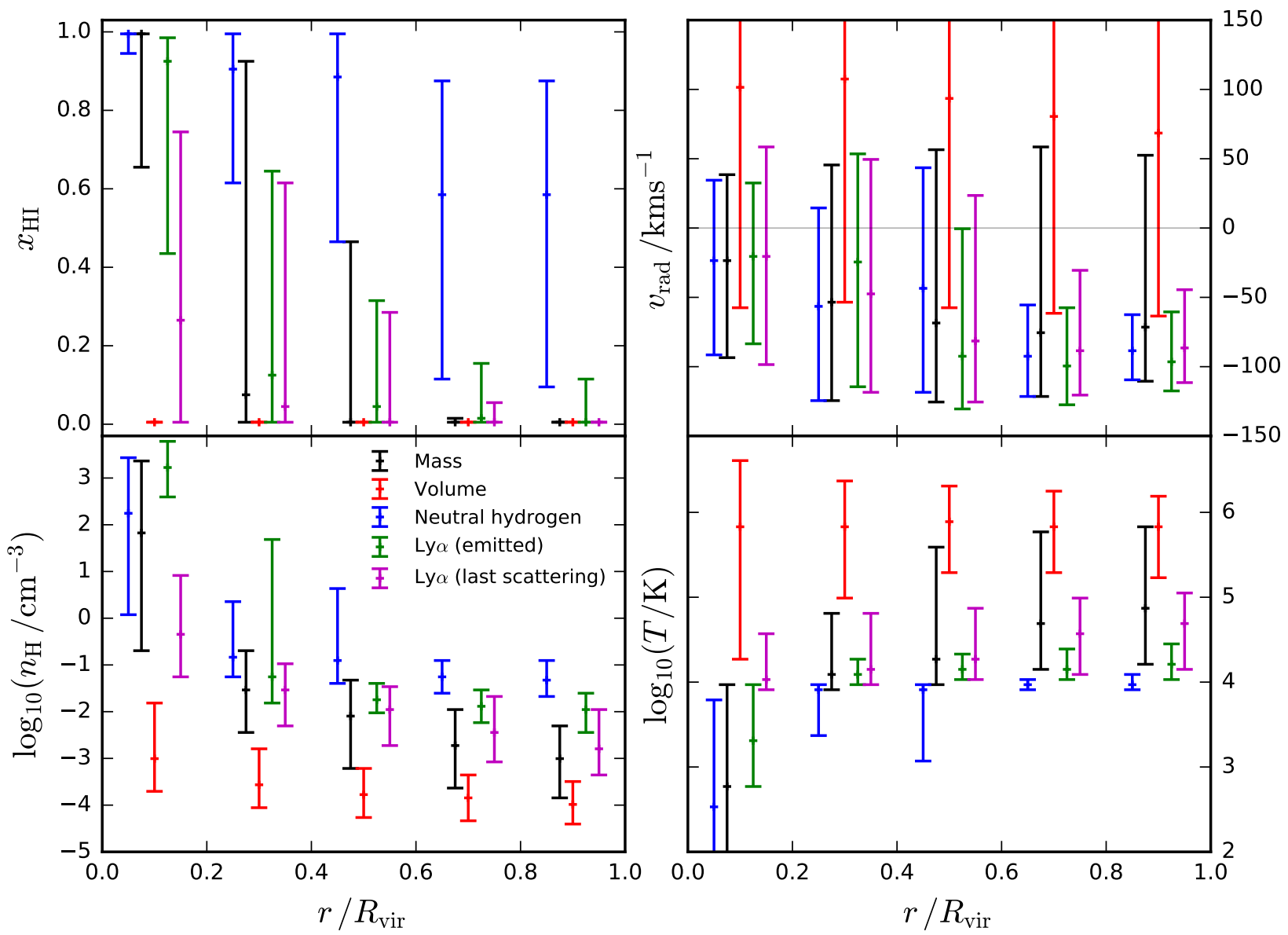

Figure 4. Distributions of neutral hydrogen fraction ( $x_{\mathrm{HI}}$, top-left), radial velocity ( $v_{\mathrm{rad}}$, top-right), hydrogen number density ( $n_{\mathrm{H}}$, bottom-left), and gas temperature ( $T$, bottom-right), all plotted as a function of radius. Gas within satellite galaxies is excluded. Points indicate the 16,50 , and $84^{\text {th }}$ percentiles of distributions weighted either by mass (black), volume (red), neutral hydrogen mass (blue), intrinsic Ly $\alpha$ luminosity (green), or the luminosity of Ly $\alpha$ photons that last interact with a given gas cell before escaping (magenta). The vast majority of escaping photons scatter at least once before escaping. Different colour points are horizontally offset from each other for plotting purposes only, and are all computed from a common set of five radial bins, ranging from $r=0$ to $r=R_{\text {vir }}$. Generally speaking, Ly $\alpha$ photons are emitted and (last) scatter from partially (or near fully) ionized and inflowing gas that is heated to slightly above $10^{4} \mathrm{~K}$. In the CGM, Ly $\alpha$ generally traces gas that is denser than the average (defined either by mass or volume), but that is less dense than the neutral hydrogen component of the CGM.

ingly dominates the neutral gas profile, although the vast majority of the hydrogen at $R_{\mathrm{vir}}$ is in an ionized phase.

The bottom panels of Fig. 3 show the connection with Ly $\alpha$ emission, splitting the luminosity profile between the same Lagrangian components. Interestingly, the intrinsic Ly $\alpha$ luminosity emitted in-situ within the CGM (lower-left panel) appears to scale with radius in a manner that is between the total and neutral hydrogen mass profiles. As with the neutral hydrogen mass profile, the intrinsic Ly $\alpha$ luminosity decreases with radius for each component. The Ly $\alpha$ luminosity declines less strongly with radius however, and the relative contribution from first-infalling gas is more reminiscent of the total mass profile. This implies that the intrinsic Ly $\alpha$ emission is not being dominated by the neutral phase (despite the higher associated densities), and a significant contribution to the total intrinsic Ly $\alpha$ luminosity of the CGM comes from warmer ionized gas, particularly in the outer CGM. Note that satellite galaxies are excluded from our analysis at this stage, which would otherwise dominate the intrinsic $\operatorname{Ly} \alpha$ luminosity outside of the central ISM (but not the escaping luminosity).
The lower-right panel of Fig. 3 focuses on escaping Ly $\alpha$ emission as a tracer of the CGM. The solid black line shows the total Ly $\alpha$ luminosity that escapes the halo after last scattering from a given radius within the CGM (or from the radius of emission if the escaping photons do not scatter). This (unlike the intrinsic CGM emission profile) therefore does include a contribution from photons that are emitted within the central ISM or within satellite galaxies, but only if those photons subsequently scatter off of circum-galactic gas. As with the other panels, the coloured lines then indicate the contribution of photons that last scatter off of different CGM components. In general, the escaping Ly $\alpha$ photons trace the CGM in much the same way as the intrinsically emitted photons shown in the lower-left panel. As a reference, the dashed black line shows the intrinsic Ly $\alpha$ luminosity of the CGM, showing that radiative transfer effects (i.e. scattering of photons emitted in the central ISM or within satellites) boost the escaping Ly $\alpha$ signal tracing the CGM out to the virial radius $(\sim 30 \mathrm{kpc})$ by roughly a factor two relative to the intrinsic profile.

The gas phases traced by $\operatorname{Ly} \alpha$ emission are shown directly in 
Fig. 4 in which distributions of gas properties are plotted, weighted by mass, volume, or associated Ly $\alpha$ luminosity. As was indicated by the shape of the radial mass/luminosity profiles shown in Fig. 3 Ly $\alpha$ generally traces partially (or fully) ionized gas that is heated to slightly above $10^{4} \mathrm{~K}$, rather than tracing the fully neutral phase directly. Ly $\alpha$ does still trace gas that is generally denser than the average within the CGM, defined either by total mass or volume weighting. Escaping Ly $\alpha$ photons (magenta points) last-scatter off gas that is warmer and lower in density than the Ly $\alpha$ emitting gas (green points) in the CGM. The vast majority of escaping photons do scatter at least once. Notably, within the ISM (far-left radial bin) escaping Ly $\alpha$ photons last-scatter from warm, partially ionized gas (median $x_{\mathrm{HI}} \approx 0.2$ ) that sits at densities above the imposed threshold for self-shielding from the UVB $\left(n_{\mathrm{H}}=0.01 \mathrm{~cm}^{-3}\right)$, highlighting the impact of photo-heating and photo-ionization from local radiation sources.

Putting the information in Fig. 3 and Fig. 4 together, we conclude that Ly $\alpha$ is a fairly faithful differential tracer of mass in the different Lagrangian components that we consider, albeit with declining luminosity as a function of radius due to the associated drop in average gas densities at larger radii. That said, our analysis so far has only considered both time and angle-averaged quantities, and in principle Ly $\alpha$ may trace specific components of the CGM preferentially if viewed from a specific direction or at a given time. This is pertinent, given that only a subset of high-redshift galaxies are observed to be LAEs, and that at least for now there is an open question in observations as to whether all rest-frame UV-visible highredshift galaxies have associated spatially extended Ly $\alpha$ haloes.

\subsubsection{Temporal variations}

Fig. 5 shows the time dependence of different spatially integrated CGM properties for both the inner CGM $\left(0.2<r / R_{\text {vir }}<0.6\right.$, left column) and the outer CGM $\left(0.6<r / R_{\text {vir }}<1\right.$, right column). The total mass in the CGM (black line, top row) is approximately constant for $z<4.5$, and generally speaking this applies to the individual Lagrangian components, with only factors of a few variations over timescales of around $100 \mathrm{Myr}$. Neutral hydrogen in the CGM varies more strongly in time (second row), particularly in the outer CGM where order of magnitude variations are seen over $100 \mathrm{Myr}$ timescales. The contribution from gas stripped (or otherwise removed) from satellites varies particularly strongly, as individual massive satellites can dominate the signal.

Strong time variations in the intrinsic Ly $\alpha$ luminosity of the inner CGM (third row, left column) are seen at high redshift $(z>$ 5 ), but subsequently variations are fairly modest, with the exception of a strong feature at $z \sim 3.6$. From visual inspection this appears to be caused by dense star-forming ISM gas briefly moving outside of $0.2 R_{\text {vir }}$ during a satellite merger. Considering also the outer CGM, the temporal variations in intrinsic Ly $\alpha$ luminosity are modest at later times, particularly when compared to the mass in neutral hydrogen.

The bottom row of Fig. 5 shows the last CGM component that Ly $\alpha$ photons interacted with before escaping the halo. The total escaping Ly $\alpha$ luminosity that traces the CGM varies only modestly in time. Short term fluctuations of about a factor two are apparent, but are partly driven by the Poisson noise in the finite distribution of Ly $\alpha$ photons used to perform Monte Carlo radiative transfer. We conclude that temporal variations are not a dominant leading-order effect for Ly $\alpha$ emission from the CGM in our simulation, at least when averaged over all angles. This has the implication that highredshift galaxies should generally be surrounded by a diffuse Ly $\alpha$ halo, irrespective of the brightness of the central Ly $\alpha$ component (which does vary strongly in time, Blaizot et al., in preparation), or even if there is no central emission component (i.e. net Ly $\alpha$ absorption against the continuum).

\subsection{The nature of observed $\operatorname{Ly} \alpha$ haloes}

Fig. 6 shows radial surface brightness profiles of escaping Ly $\alpha$ photons, now plotted as a function of projected circular radius (relative to the direction of each escaping photon). The profile is mean stacked over redshifts $3<z<4$, and over all possible viewing angles. In the top panel, we split the escaping Ly $\alpha$ photons based on the Lagrangian CGM component that each photon last interacts with before escape, using the Lagrangian components described in Section 2.3 In addition to the CGM components discussed previously, we now also show the contribution from photons that last scattered on the central ISM (orange lines, defined here as $r<0.2 R_{\mathrm{vir}}$, where $r$ in this case is the three-dimensional radius), or last-scattered within satellites (khaki lines), without interacting with the intervening CGM.

While it is expected that the central ISM dominates the production of photons observed near the centre of the projected profile, it is notable that the majority of the photons observed in the central region of the profile $\left(r_{\text {projected }}<7 \mathrm{kpc}\right)$ do not scatter in the CGM (orange line). At larger separations, the two main contributors to the signal are the "first infall" gaseous component (blue line) and the component of photons that escape from satellite galaxies (khaki line). If the simulation picture is correct, this implies that observed Ly $\alpha$ haloes are tracing primarily inflowing circumgalactic gas along with emission escaping from satellites (at least when stacked).

The lower panel of Fig. 6 splits the escaping Ly $\alpha$ emission based on the original source of the emission (i.e. before scattering), splitting between the ISM of the central galaxy (dashed lines), the ISM of satellites (dotted), and in-situ photons emitted within the CGM (dash-dotted). The largest contributor at projected separations $<10 \mathrm{kpc}$ is from photons that were emitted within the ISM of the central galaxy (note that this is before any point spread function convolution is applied). At larger separations the escaping radiation is comprised of a roughly equal mix of scattered photons from the central ISM, in-situ CGM photons, and photons emitted within satellites ${ }^{4}$ Over $10<r_{\text {projected }} / \mathrm{kpc}<20$ the in-situ component provides marginally the largest contribution, with satellites increasingly powering the profile at larger separations (forming an effective "two-halo term" outside the halo virial radius, see also Zheng et al. 2011, Mas-Ribas \& Dijkstra 2016, Mas-Ribas et al.2017), though at these radii the stacked surface brightness has dropped significantly below the $\sim 10^{-19} \mathrm{ergs}^{-1} \mathrm{~cm}^{-2} \operatorname{arcsec}^{-2}$ limit of non-stacked MUSE deep-field observations of individual LAEs (e.g. Wisotzki et al. 2016, Leclercq et al. 2017). The transition with projected separation from central-dominated, to diffuse CGM, to faint satellites setting the SB profile is consistent with the simulations-based study of Lake et al. (2015), who simulate a brighter LAE at the same redshifts. Note also that after convolving with the MUSE PSF, the central ISM component is pushed outwards, and contributes comparably to the other components out to $\sim 25 \mathrm{kpc}$ in projection.

\footnotetext{
4 As a reminder, we refer to both satellite subhaloes (within the virial radius of the primary host halo) and neighbouring haloes (outside the virial radius of the primary host halo) as "satellites".
} 

$0.2<r / R_{\text {vir }}<0.6$
$0.6<r / R_{\text {vir }}<1$
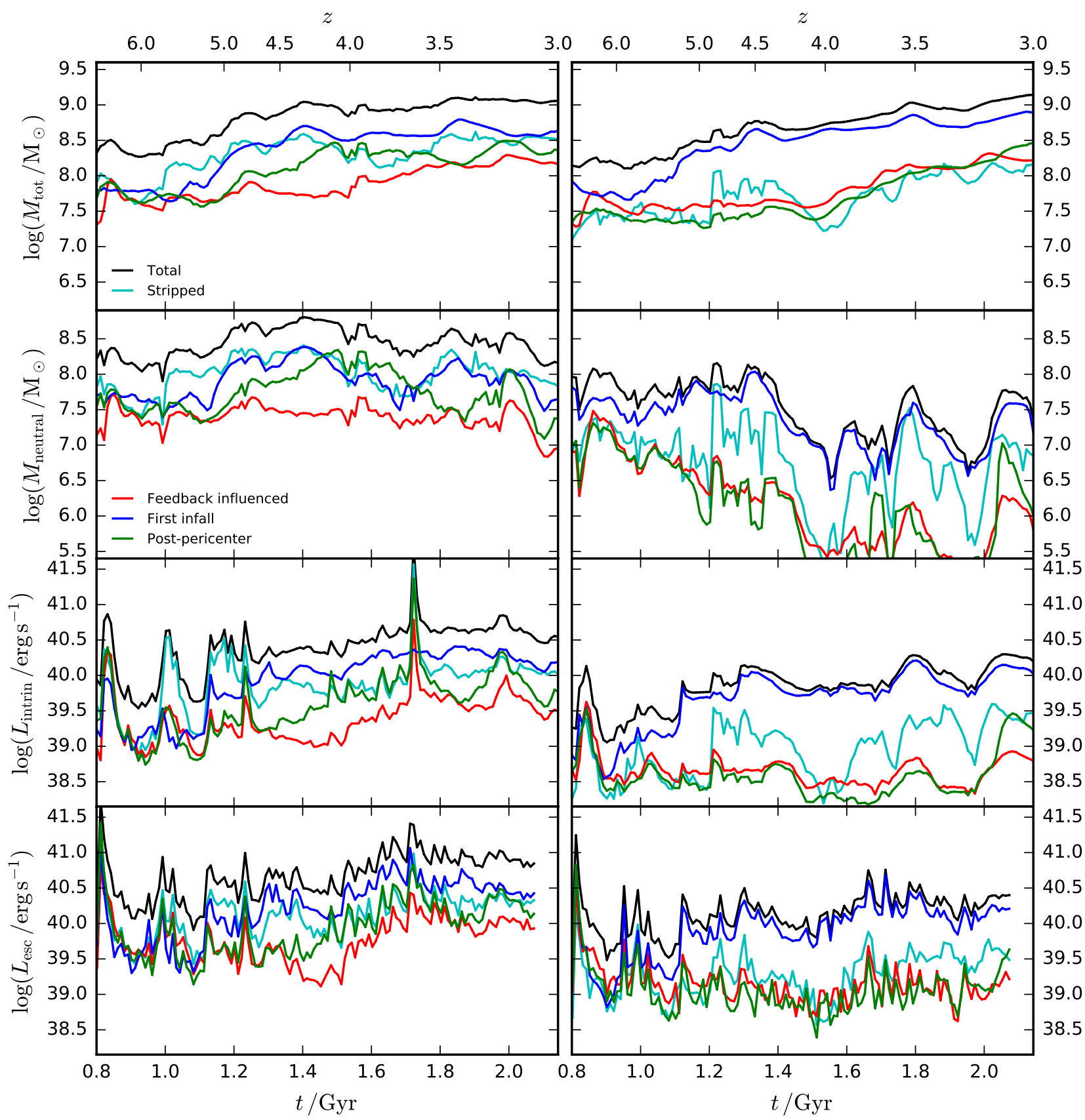

Figure 5. Time evolution in mass/luminosity of different CGM components (excluding satellite galaxies). Different rows show total gas mass (top row), neutral hydrogen mass (second row), intrinsic Ly $\alpha$ luminosity (third row), and escaping Ly $\alpha$ luminosity (bottom). The left column shows the inner CGM over $0.2<r / R_{\mathrm{vir}}<0.6$, and the right column shows the outer CGM over $0.6<r / R_{\mathrm{vir}}<1$. Generally speaking, temporal fluctuations are relatively modest after $z=5$; with escaping Ly $\alpha$ luminosity tracing the CGM only varying by factors of a few over short timescales.

An important outstanding question for the interpretation of observed Ly $\alpha$ haloes is the nature of the powering mechanism for the emission, which can be scattering of Ly $\alpha$ photons escaping the ISM, emission associated with satellite galaxies (which are likely unresolved even in deep observations of LAEs), in-situ emission powered by ionizing UV radiation escaping the galaxy or from the UVB, or alternatively in-situ emission powered by compressive heating associated with gravitational infall or feedback processes. While we have addressed the role of scattering and satellite galaxies here, the powering of the in-situ CGM emission is not trivially known even in a simulation. It is sometimes assumed that collisional excitations in the CGM are predominantly powered by grav- 


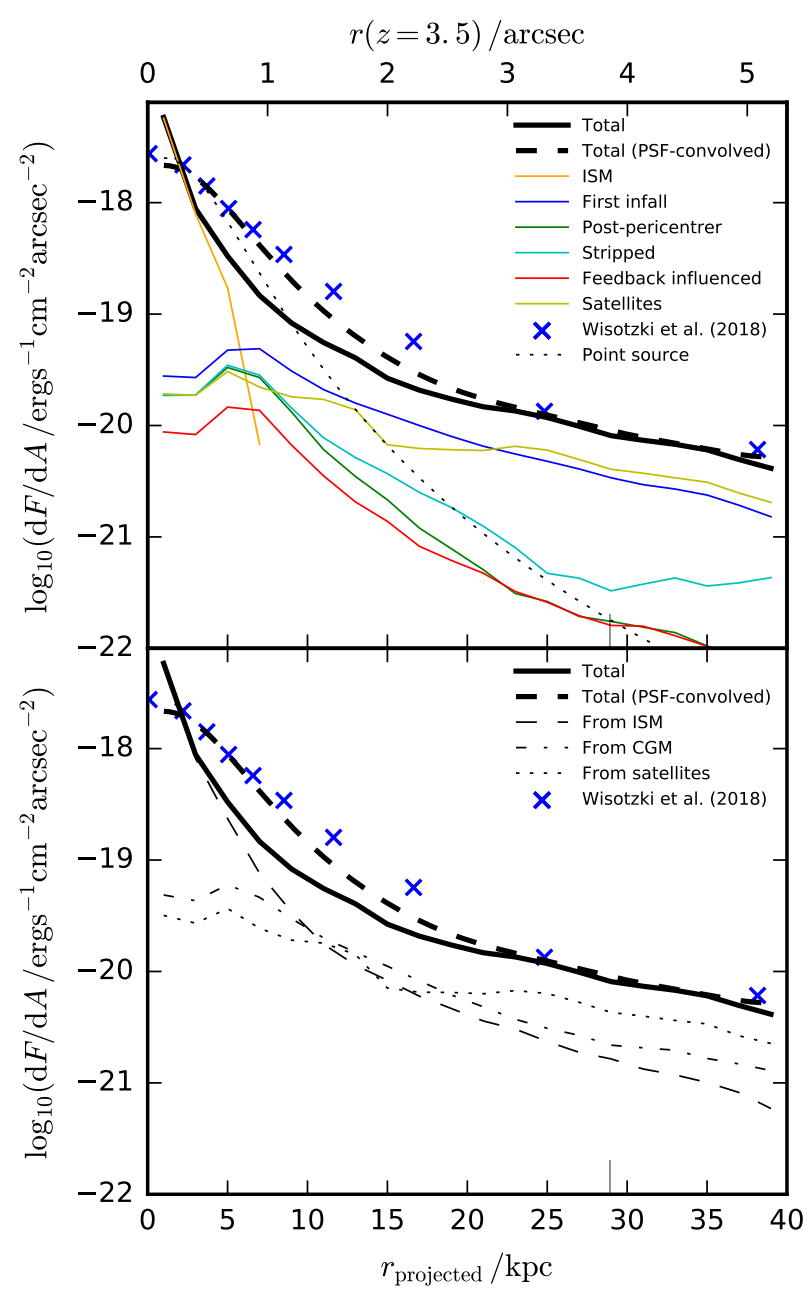

Figure 6. Mean stacked $(3<z<4)$ and angle-averaged Ly $\alpha$ surface brightness profiles, plotted as a function of projected separation from the halo center. The thick solid black line in both panels shows the total escaping Ly $\alpha$ surface brightness profile from the simulation. The thick dashed black line shows the same profile after convolving with the typical point spread function (PSF) inferred for deep-field MUSE observations. For reference, the dotted line in the top panel shows the PSF-convolved profile of a scaled point source. The virial radius of the simulated halo at $z=3.5$ is $R_{\mathrm{vir}}=29 \mathrm{kpc}$, as marked by a small grey indicator at the bottom of each panel. In the top panel, thin coloured lines show how escaping Ly $\alpha$ emission traces different components of the CGM, with each photon associated with the component it last interacted with before escape. For the projected profiles shown here, we now also include photons that escape from the central ISM (orange line), and from satellite galaxies (khaki line), without interacting with the intervening CGM. In the bottom panel, thin lines show the origin of escaping Ly $\alpha$ emission, indicating whether escaping photons at a given projected radius were originally emitted within the central ISM (dashed line), in situ within the CGM (dash-dotted), or within a satellite galaxy (dotted). For comparison, the stacked Ly $\alpha$ surface brightness profile of 92 detected Ly $\alpha$ emitters from the MUSE HDFS and UDF fields is shown by blue crosses, taken from Wisotzki et al. (2018).

itational heating, and that recombinations in the CGM are powered by fluorescence, but in practice compressive heating of dense gas will lead to recombinations (though admittedly we expect photoionization to be the dominant ionization mechanism) and photoheating from UV photons will lead to collisional excitations.

We leave the task of separating these effects for future work,

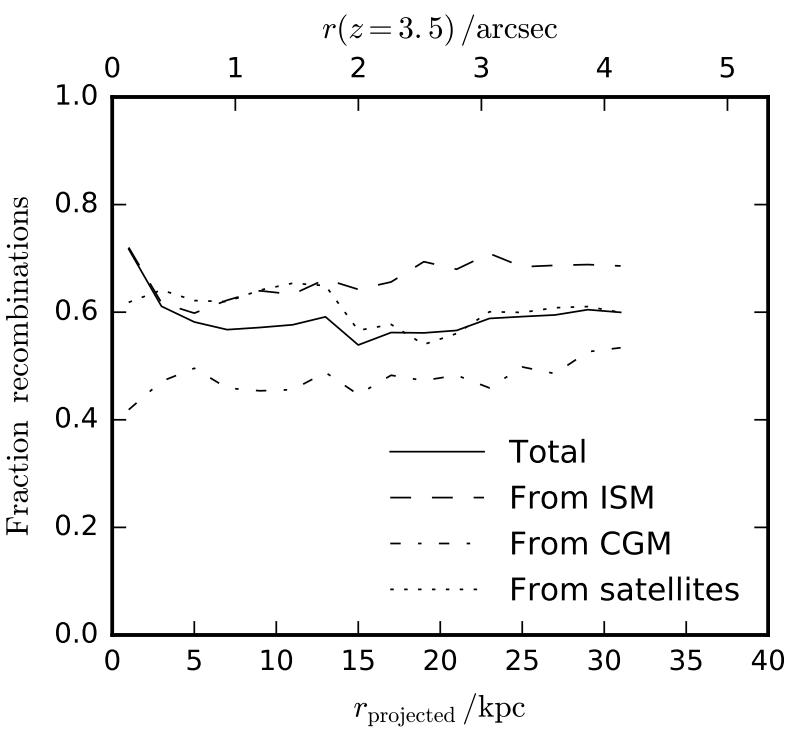

Figure 7. The fraction of the projected Ly $\alpha$ surface brightness contributed by photons produced in recombinations (as opposed to collisional excitations), plotted as a function of projected separation from the halo center. As in Fig. 6 data are taken from a mean, angle-averaged stack of our simulated galaxy over $3<z<4$ (though note that no PSF convolution is applied here). Lines show the fractional contribution of recombinations for the total surface brightness profile (solid line), and for photons that were originally emitted inside the ISM (dashed), inside satellite galaxies (dotted), and insitu within the CGM (dash-dotted). Collisional excitations provide a fractionally larger contribution to the escaping Ly $\alpha$ flux produced in-situ within the CGM $(\approx 50 \%)$, whereas recombinations contribute the majority of the escaping flux for photons emitted within the ISM $(\approx 65 \%)$. Recombinations generally contribute $\approx 60 \%$ of the total escaping flux.

but we do consider the relative contribution of recombinations versus collisional excitations in Fig. 7 Recombinations provide the majority of escaping photons that were emitted within the ISM (about $65 \%$ ), but collisional excitations provide around $50 \%$ for the in-situ CGM component. This implies that different physical processes are likely powering the in-situ CGM emission relative to the ISM emission.

\subsection{Comparison to surface brightness profiles from stacked MUSE observations}

Fig. 6also compares our stacked simulation profile with the stacked Ly $\alpha$ surface brightness profiles measured using MUSE observations of $923<z<4$ LAEs from the Hubble Deep field South, and the Hubble Ultra Deep Field (Wisotzki et al.2018). The thick soliddashed line in Fig. 6 shows our simulation prediction after convolving with the appropriate point spread function (PSF). We adopt a Moffat profile with $\beta=2.8$, and FWHM $=0.875-\frac{1}{3}\left(\lambda / 10^{4} \AA\right)$, as inferred for the relevant deep-field MUSE observations (Bacon et al.2017). For reference, we also show the profile of a scaled point source, convolved with the same PSF. As discussed in Wisotzki et al. (2016 2018), MUSE clearly resolves spatially extended emission outside of the central projected arcsecond.

After PSF convolution the agreement between the observed and simulated stacked profiles is generally excellent, though perhaps partially fortuitous given that we have only simulated a single galaxy. The agreement in shape within the inner $\sim 7 \mathrm{kpc}$ in projection is not necessarily physically meaningful, given that the profile 
on these scales is dominated by PSF-broadening (comparison to the dotted line, top panel). For larger scales where PSF-broadening is sub-dominant, the simulated profile under-predicts the observed profile by up to $50 \%$ for the range $10<r_{\text {projected }} / \mathrm{kpc}<20$, which may be significant given that this is the range where we predict in-situ CGM emission to play the largest role. This could be interpreted as evidence that we have insufficient dense and/or neutral gas in the CGM of our simulated halo, possibly because our simulated galaxy is too low in halo mass (we believe our simulated galaxy has a stellar mass which is too large relative to its halo mass, see discussion in Section 4.2, or possibly because we are missing a dense/neutral phase associated with supernova-driven outflows. For yet larger separations $\left(r_{\text {projected }} / \mathrm{kpc}>20\right)$ close to and beyond the halo virial radius, agreement with the observations is excellent. Overall, the level of agreement with observations is encouraging, and inspires a level of confidence in our results regarding the origin of observed Ly $\alpha$ haloes (though see the forthcoming discussing on Ly $\alpha$ spectral morphologies).

Note that the simulated surface brightness profile does not exactly follow the observational selection and stacking procedure. Specifically, the simulated profile shown here is mean stacked and uses angle-averaged Ly $\alpha$ outputs. This is necessary in order to split photons into different component $5^{5}$ The observational profile is instead median stacked, with the motivation being to prevent faint undetected companions from enhancing the signal in the profile outskirts. In our simulation, we resolve $\approx 100$ satellites per projected annular bin of width 0.5 arcsec, of which there are generally $\approx 20$ satellites that contain stars (see, e.g., figure 2 in Mitchell et al. 2018, for a visual impression). When looking at radial profiles (not in projection) of escaping Ly $\alpha$ luminosity, we therefore find that while median (as opposed to mean) stacking does reduce the contribution from satellites in the average profile (by effectively excluding the brightest satellites), the reduction is modest (roughly a factor two in the number of the escaping photons that escape from satellites, and a $25 \%$ reduction in the number of escaping photons that were originally emitted within satellites before scattering). The contribution from diffuse inflowing gas is insensitive to mean versus median stacking, and is therefore slightly up-weighted in relative importance for a median stack (compared to the mean stack shown in Fig. 6.

A more realistic comparison to the observed profile is shown in Appendix A using mock data cubes orientated along specific lines of sight (rather than angle-averaging), median stacking (matching the observed procedure), and an explicit flux selection (rather than simply averaging all simulations outputs over $3<z<4$ ). The more realistically produced profile almost exactly resembles the simulation profile shown in Fig. 6

\subsection{Ly $\alpha$ spectral morphology}

Fig. 8 shows the angle-averaged Ly $\alpha$ spectrum of escaping photons for our simulation, mean stacked over $3<z<4$. Since we are considering angle-averaged spectra, each Ly $\alpha$ photon is adjusted in frequency to account for the peculiar velocity of the halo within the cosmological box (otherwise the stacked spectrum would be artificially broadened). The top panel of Fig. 8 shows

\footnotetext{
5 Median stacking requires the production of direction-dependent mock data cubes, which in turn requires the use of the peeling-off technique to obtain adequate sample statistics. With peeling-off we lose information about the origin of individual photons however.
}

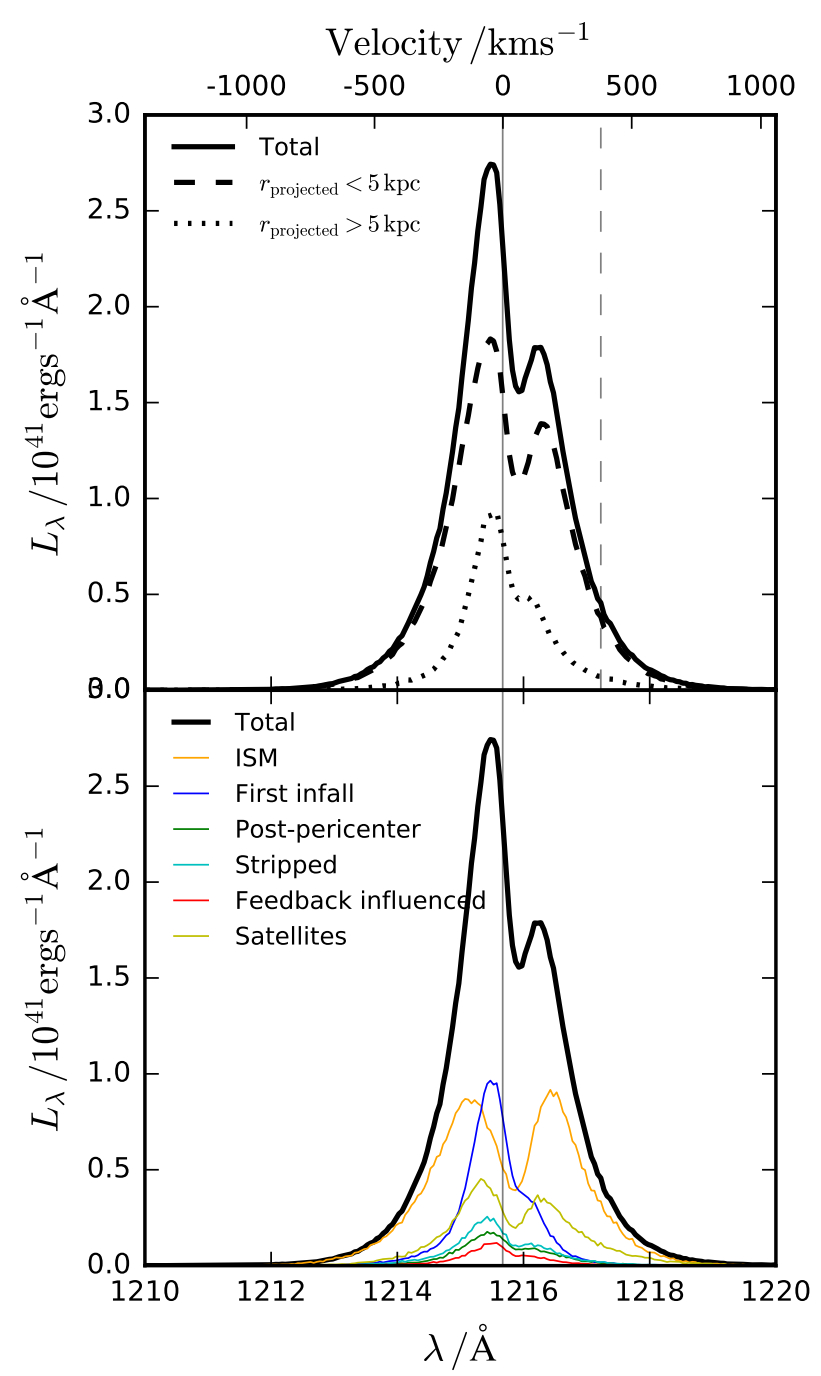

Figure 8. Stacked $(3<z<4)$, angle-averaged spectra of escaping Ly $\alpha$ photons. The top panel shows the relative spectral morphology of the inner versus outer region, split at $5 \mathrm{kpc}$ in projection. The bottom panel shows the contribution of different components, splitting photons by which galaxy/CGM component each photon last interacted with before escaping, as indicated by the legend. The dashed grey vertical line shown in the top panel indicates the peak shift of the Ly $\alpha$ line predicted by the empirical relation from Verhamme et al. (2018), given the full width at half maximum of our simulated spectrum for $r_{\text {projected }}<5 \mathrm{kpc}$. Contrary to observed LAEs at $z \sim 3$ (for which IGM attenuation is expected to be modest), our simulated spectrum exhibits flux bluewards of the systemic velocity that is comparable to the flux redwards of systemic. As a consequence, the red peak of the simulated (inner region) spectrum is insufficiently redshifted relative to systemic, given the FWHM and the Verhamme et al. (2018) relation.

the dichotomy between the spectrum of the spatial centre in projection $\left(r_{\text {projected }}<5 \mathrm{kpc}\right.$ ), and the spectrum of the spatially extended component. Most of the flux escapes from the central component, exhibiting a double peaked spectral morphology, with a dominant blue peak slightly bluewards of the systemic velocity, and a more offset and a subdominant red peak. In contrast, the spatially extended component is asymmetric, single peaked, and peaking slightly bluewards of systemic.

The lower panel of Fig. 8 shows the relative contribution of different Lagrangian components to the overall profile (divided on 
the basis of which component each escaping photon last interacts with before escaping the system). The spectral morphology of the flux tracing the central ISM (orange line) is the broadest component, reflecting presumably the higher neutral HI optical depth of the dense ISM, thus requiring larger frequency excursions before escape (though there is still significant flux escaping at the systemic velocity, implying the existence of copious low-column density sight lines). The central ISM component is double-peaked, and near-symmetric around systemic. Ly $\alpha$ escaping from satellites (khaki line) is also double-peaked, but is less symmetric. Satellites are responsible for the slight red "bump" feature that is apparent for the spatially extended ( $r_{\text {projected }}>5 \mathrm{kpc}$ ) component in the top panel. All of the other CGM components in the lower panel are slightly blue-shifted and single peaked, explaining the overall elevation of blue relative to red flux in the total spectrum shown in the top panel.

For the CGM-tracing components, an overall blueshift is expected for an inflowing medium (e.g. Dijkstra et al. 2006, Verhamme et al.2006), albeit with a fairly modest kinematic shift due to the smaller expected absolute radial velocities of inflows compared to outflows. Typical radial inflow velocities in our simulation are of order 50 to $100 \mathrm{kms}^{-1}$ (see Fig. 4). It is notable that the component tracing the gas affected by stellar feedback (red line) is not clearly redshifted, given that feedback-driven outflows are commonly invoked to explain the systematically redshifted spectral morphology of observed LAEs (e.g. Ahn 2004, Verhamme et al. 2006). Note that our definition of the "feedback-influenced" component includes gas that was affected by stellar feedback at any time in the past however, and is therefore comprised of a mix of both inflowing and outflowing gas. Nonetheless, the lack of a strong redshifted component reflects that fast-moving outflows in our simulation are generally fully ionized (see, e.g., Fig. 2 and Fig. 4), and are therefore transparent to Ly $\alpha$ radiation.

Our simulated spectrum does not resemble the typical Ly $\alpha$ spectrum of observed high-redshift galaxies. Observed Ly $\alpha$ spectra (usually measured in an aperture that is comparable in size to the central ISM) are nearly always observed with a red peak that is substantially redshifted relative to systemic, are asymmetric with an extended tail towards the red, occasionally show a secondary blue peak, and often show a strong deficit of photons at the systemic redshift if the secondary peak is present (e.g. Shapley et al. 2003 . Tapken et al. 2007, Kulas et al. 2012, Trainor et al. 2015). There are observed counter-examples: for example a LAE with a double peak, a dominant blue peak and significant emission at the systemic velocity was reported recently by Erb et al. (2019), and objects similar to our stacked spectrum (i.e. are more symmetric around the systemic velocity) are occasionally seen (e.g. Tapken et al. 2007, Erb et al. 2016, but are not representative. Recently, Hayes et al. (2020) presented mean and median stacks of LAEs from MUSE observations (albeit for LAEs that are generally brighter than the object studied here), demonstrating that any flux bluewards of systemic is completely subdominant to a dominant redshifted component. Furthermore, using observed Ly $\alpha$ absorption statistics, they find that the intervening IGM has a modest $\approx 25 \%$ attenuation of emission bluewards of the systemic velocity at $z \sim 3$, and furthermore demonstrate that this is consistent with a comparison with low-z LAEs, for which IGM attenuation is thought to be negligible. Simulation-based studies have reported slightly stronger IGM attenuation at $z \sim 3$ however (e.g. Byrohl \& Gronke 2020).

Verhamme et al. (2018) show that a positive correlation exists between the Ly $\alpha$ FWHM, and the velocity shift of peak from the systemic redshift of the galaxy. Given the FWHM of our stacked spectrum, we plot the predicted $\operatorname{Ly} \alpha$ peak velocity $\left(V_{\text {peak }} \approx\right.$ $400 \mathrm{kms}^{-1}$ ) as the dashed grey vertical line in the top panel of Fig. 8 While there is systematic scatter in the Verhamme et al. (2018) empirical relation, this serves to underline that even for the ISM-tracing component, we likely significantly underestimate the velocity shift of the red peak in the spectrum.

Another consideration is the comparison of the Ly $\alpha$ spectral morphology between the spatial center and the spatially extended halo. Recent spatially resolved observations of high-redshift Ly $\alpha$ spectra reveal significant diversity in Ly $\alpha$ halo spectra (the halo can be both red or blue-shifted with respect to the central region), but that the halo spectra are still significantly redshifted from the systemic velocity of the system (Swinbank et al. 2007), Smit et al. 2017, Claeyssens et al. 2019, Leclercq et al. 2020), although Erb et al. (2018) present a more complex case where a double-peaked morphology is seen in the outer halo. These observations indicate that the unrealistic spectral morphology of our simulated galaxy is therefore not likely confined to a problem on scales within the central ISM alone, though it is still conceivable that the ISM is where large velocity shifts are imparted if most of the extended emission is scattered from the CGM after being emitted within the ISM.

While it is possible that our simulated galaxy is an outlier in some way relative to the broader population, the differences between (typical) observed spectra with our simulation more likely imply that we do not have sufficient HI column densities, and that we are missing a strongly outflowing and volume-filling neutral gas component in our simulation. We note that if we inspect viewing angle-specific spectra (using the peeling off technique) instead of angle-averaged spectra, we do see a very significant diversity in the spectral morphologies (even if viewed from different angles at a single redshift), including rare cases where there is a dominant redshifted component. We do not however find examples of spectra with negligible escaping emission bluewards of the systemic velocity, reinforcing the tension between our stacked angle-averaged spectrum and observations.

\section{DISCUSSION}

\subsection{Ly $\alpha$ spectra from cosmological simulations}

Given that our simulation fails on average to produce the characteristic single peaked, redshifted from systemic, and asymmetric spectral profile characteristic of observed LAEs at $z \sim 3$, we here review results presented from other cosmological simulations in the literature, to assess the generality of the problem with simulations. We focus on analyses of simulations that attempt Ly $\alpha$ radiative transfer within the ISM, and that account (with varying degrees of sophistication) for ionizing UV radiation from local stellar sources.

Smith et al. (2019) present an analysis of a single cosmological zoom-in simulation from the FIRE simulation suite (Hopkins et al. 2014) for redshifts $z>5$, with comparable resolution to the simulation presented here. Their figure 11 shows that before IGM attenuation is applied, their spectra also show strong blue peaks, and also high flux levels at the systemic velocity between the two peaks, similar to the ISM-tracing component for our galaxy shown in Fig. 8 As they focus on higher redshifts, they are able to achieve reasonable looking single-peaked redshifted Ly $\alpha$ spectra after IGM attenuation is applied, as the IGM attenuates almost all of the flux at line centre and on the blue side. IGM attenuation is not likely to be a sufficient explanation at lower redshifts however (e.g. Dijkstra et al. 2007, Laursen et al. 2011, Inoue et al. 2014, Hayes et al. 
2020), and it would be interesting to see analysis of the Ly $\alpha$ spectra from the FIRE simulations presented at lower redshifts.

Behrens et al. (2019) analyse a single cosmological zoom-in simulation run with the RAMSES code (without radiation hydrodynamics). Again, as their analysis is presented at $z=7$ the IGM completely attenuates the Ly $\alpha$ spectrum both at, and bluewards of the systemic velocity. Before their IGM correction their spectra appear dominated by emission from a blue peak. Laursen \& SommerLarsen (2007), Laursen et al. (2009a) and Laursen et al. (2009b) present analyses of an older and lower resolution (gas particle mass $\sim 10^{5} \mathrm{M}_{\odot}$ ) cosmological zoom-in simulation of a set of galaxies at $z=3.6$, and find similar results to Smith et al. (2019), in that the escaping Ly $\alpha$ spectrum is double-peaked, with (in most cases) a stronger blue peak, and with substantial flux emerging at the systemic velocity.

As a counter-example, while not a live cosmological simulation, Verhamme et al. (2012) analysed the emerging Ly $\alpha$ spectra from two idealised low-mass disk galaxies. When the ISM was allowed to cool radiatively below $10^{4} \mathrm{~K}$ (their "G2" simulation), the emerging Ly $\alpha$ profile is double-peaked when viewed edge-on, but is asymmetric when viewed face-on, and with a single peak that is located redwards of the systemic velocity, as is typical in real observations. This is attributed to outflowing gas in the simulation, but it is not clear how the outflows in their simulation compare to the full cosmological simulations discussed previously. A second counterexample is provided by the recent analysis of a comparatively lowresolution cosmological simulation by Chung et al. (2019), who do find redshifted spectra. In this case however, they utilise a subgrid feedback model that temporarily decouples "wind" particles from the hydrodynamical scheme, and ejects these particles from the ISM at high velocity. In such a scheme, supernova-driven outflows can remain in a neutral phase even while outflowing at many hundreds of $\mathrm{kms}^{-1}$, presumably explaining the difference in the predicted spectral morphology with the other cosmological simulations discussed.

It can be argued that cosmological simulations produce unrealistic Ly $\alpha$ spectral morphologies because they do not sufficiently resolve the internal structure of star-forming clouds within the ISM, where many of the photons are produced (in particular the Stromgren radius and turbulent structure will be under-resolved). Using idealised radiation hydrodynamics simulations at $\sim 0.25 \mathrm{pc}$ resolution (a factor 50 higher than for the cosmological simulation studied here), Kimm et al. (2019) study the escape of Ly $\alpha$ photons from individual star-forming clouds, isolated from the effects of the surrounding diffuse ISM and CGM. They find that radiation feedback from young stars can drive cloud expansion and so create red-peak dominated Ly $\alpha$ spectra, though they also show that if star formation efficiencies are high $(10 \%)$ clouds are disrupted early, leading to luminosity-weighted, time integrated $\operatorname{Ly} \alpha$ spectra that are narrow and not kinematically shifted. Kimm et al. (2019) also find that they not succeed in reproducing the large velocity shifts of the red peak that are often observed, concluding that larger-scale radiation transfer effects in the ISM/CGM are required.

Putting all of these examples together, we conclude that we have yet to see clear evidence that current state-of-the-art cosmological simulations are capable of producing realistic Ly $\alpha$ spectra, unless wind particles are explicitly decoupled from the hydrodynamical scheme. This point has also been discussed by Gronke et al. (2017), who interpret this problem as evidence that simulations indeed are not resolving an important population of tiny optically thick clumps, concluding that sub-resolution models for unresolved clumping in the ISM/CGM should be implemented when post-processing cosmological simulations with Ly $\alpha$ radiative transfer. The relative lack of spatial resolution in the CGM has in particular received a great deal of attention recently (Hummels et al. 2019. Peeples et al. 2019, Suresh et al. 2019, van de Voort et al. 2019), and may be particularly pertinent given that we find that most of the escaping Ly $\alpha$ radiation from our galaxy does not scatter from the CGM (orange line, top panel, Fig. 6). Another aspect that has received attention is the potential for cosmic rays to play an important role in regulating escaping Ly $\alpha$ spectra (Gronke et al. 2018), as cosmic rays can be more resistant to dissipative losses and so more smoothly distribute the injected feedback energy and momentum (both spatially and temporally), leading to less impulsive but more persistent galactic-scale outflows that are better able to entrain and maintain neutral hydrogen clouds.

At the same time, the unrealistic spectral morphology of our simulated galaxy needs to be reconciled against the realistic Ly $\alpha$ surface brightness profile, which closely reproduces stacked MUSE observations (Fig. 6 and Fig. A1). At face value, adding copious amounts of outflowing neutral gas to the CGM of our simulation could lead to an over-production of Ly $\alpha$ surface brightness at larger projected separations. We do however under-predict the surface brightness at projected separations of $\sim 15 \mathrm{kpc}$ by about $50 \%$, leaving room to add outflowing neutral gas at these scales to act as a scattering medium. Recently, Song et al. (2020) have demonstrated that it is possible to reproduce both the surface brightness profiles and spectral morphology of MUSE-observed LAEs, using idealised spherically-symmetric models that feature a continuous expanding medium extending outwards to scales of $\sim 20 \mathrm{kpc}$ with outflowing velocities $\sim 200 \mathrm{kms}^{-1}$ (their table 3 ). In future work we plan to see if similar results can be obtained empirically by modifying our simulations in post-processing such that they they contain trace amounts of neutral hydrogen in diffuse outflowing winds.

\subsection{Producing realistic stellar distributions in simulations of high-redshift galaxies}

An important caveats in our comparison to observed $\operatorname{Ly} \alpha$ surface brightness profiles is the question of whether we are simulating a LAE with the correct halo mass (see for example Rosdahl \& Blaizot 2012, who predict that the in-situ Ly $\alpha$ luminosity of the CGM strongly correlates with halo mass). The top panel of Fig. 9 shows the stellar mass of our simulated galaxy (red point at $M_{200}=10^{11.1} \mathrm{M}_{\odot}$ ), compared to extrapolations of empirical constraints from Behroozi et al. (2013) and Moster et al. (2013). With a stellar mass of $M_{\star}=10^{9.5} \mathrm{M}_{\odot}$, our simulated galaxy has an uncomfortably high stellar mass given its halo mass, sitting an order of magnitude above the extrapolated best-fit empirical relations.

We also show the same galaxy but run without radiation hydrodynamics, both for the case of no stellar feedback (magenta points) and with supernova feedback using a fixed time delay of $10 \mathrm{Myr}$ for supernova explosions (black points). As a reminder, the fiducial RHD simulation used in this study includes radiation feedback from local stellar sources, boosts the injected supernova momentum to account for unresolved photo-heating (Geen et al. 2015), and uses a more realistic time delay distribution function for supernova explosions. Excluding the boost associated with unresolved photo-heating, both the $\mathrm{RhD}$ and non-RhD simulations with feedback inject four times the supernova energy expected for a Kroupa IMF (see Section 2.1.4). While the RHD simulation forms about half the mass in stars compared to the no-feedback case, it forms twice as many stars as the pure hydrodynamics simulation with a fixed time delay. Evidently the expected reduction in stars 

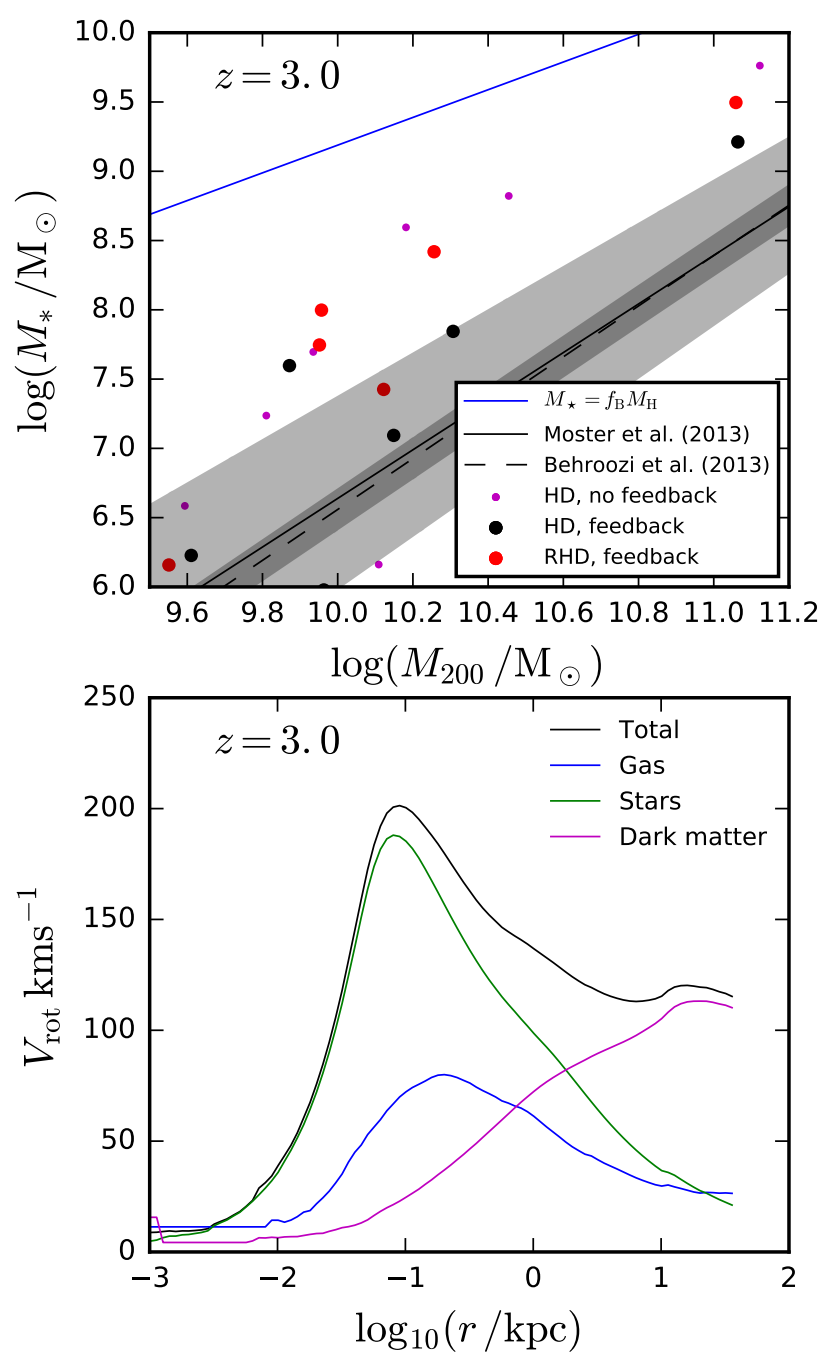

Figure 9. Basic properties of our simulated galaxy. Top: stellar mass as a function of dark matter halo mass, plotted at $z=3$. Red points show galaxies from the fiducial RHD simulation used in this study (which uses a realistic time delay distribution for supernova explosions). Magenta points show galaxies from a hydrodynamics-only simulation, performed without stellar feedback, using the same initial conditions (Mitchell et al. 2018). Black points show galaxies from a corresponding hydrodynamics-only simulation that includes stellar feedback, using a fixed time delay of $10 \mathrm{Myr}$ for supernova explosions. The main target galaxy is the most massive galaxy in each simulation with $M_{200} \sim 10^{11.1} \mathrm{M}_{\odot}$ (other points are from neighbouring lower-mass galaxies within the zoom-in region). Extrapolated constraints from abundance matching are shown by the grey lines bands, and are taken from Behroozi et al. (2013) and Moster et al. (2013). The blue diagonal line indicates the point where stellar mass would be equivalent to halo mass multiplied by the cosmic baryon fraction. Our simulated galaxy is uncomfortably high in stellar mass given the halo mass, with any reduction in star formation from including RHD seemingly more than offset by using a more realistic time delay distribution function for supernova explosions (less temporally clustered supernovae apparently result in higher radiative losses). Bottom: Rotation curve ( $\left.V_{\text {rot }} \equiv G M(r) / r\right)$ of the main galaxy from our fiducial RHD simulation at $z=3$, decomposed into the contribution from stars, gas and dark matter. The rotation curve shows that the stellar distribution is highly centrally concentrated, indicative of likely radiative overcooling. formed due to increased momentum input plus radiation feedback is more than offset by reducing the temporal clustering of supernova explosions, which presumably increases the radiative cooling losses (somewhat contrary to expectations from recent work on idealised simulations, e.g., Gentry et al. 2020, Keller \& Kruijssen 2020).

In addition, the lower panel of Fig. 9 shows the galaxy rotation curve (assuming spherical symmetry), decomposed into contributions from stars, gas, and dark matter. The stellar component is highly centrally concentrated, such that the resulting rotation curve is far from flat. While such rotation curves cannot be currently ruled out for faint galaxies at $z>3$, we nonetheless interpret this as an additional sign that the simulation likely suffers from overly high radiative losses relative to the energy injected by feedback. We note that at this halo mass we are on the cusp of the regime where feedback from a supermassive black hole may start to become relevant (Dubois et al. 2015), which may be an important missing component needed to prevent the formation of such a compact stellar core (and could in principle also act as a mechanism to accelerate dense gas to the velocities required to explain observed Ly $\alpha$ spectral morphologies).

If it is the case that our galaxy has a stellar mass that is overly large relative to its halo mass, the implication would be that the relative contribution of photo-heating from local stellar sources to Ly $\alpha$ emission could be over-estimated relative to gravitional heating, and relative to heating from the wider UVB. This is not guaranteed however, since more efficient stellar feedback (needed to reduce the stellar mass) would also reduce the masses of neutral atomic Hydrogen and dust within the ISM, feasibly increasing Ly $\alpha$ escape fractions for centrally emitted photons (which can then scatter from the CGM). Furthermore, since galaxy stellar mass scales approximately as halo mass squared in the halo mass range of our simulated galaxy (e.g. Behroozi et al. 2013, Moster et al. 2013), a simulation with more efficient feedback would only require a relatively modest change in halo mass to recover the same stellar mass (and therefore overall Ly $\alpha$ brightness). As such, we do not expect the predicted contribution of gravitional heating to Ly $\alpha$ production (which is expected to scale linearly with halo mass, Dijkstra \& Loeb 2009, Rosdahl \& Blaizot 2012) to be strongly sensitive to the efficiency of feedback in our simulation.

\section{SUMMARY}

In this study we set out to explore the origin of dense gas in the circum-galactic medium (CGM) at high-redshift $(z \sim 3)$, and the implications for spatially extended Ly $\alpha$ emission from the CGM. We have presented results taken from a radiation hydrodynamics simulation of a high-redshift galaxy with a halo mass of $\sim 10^{11} \mathrm{M}_{\odot}$ at $z=3$.

We find that the spherically averaged radial mass profile of circum-galactic neutral hydrogen in the simulation drops strongly with radius (Fig. 1), and that this profile is shaped primarily by the compression of cosmologically infalling gas pushing the typical hydrogen number density above the threshold for efficient self-shielding from ionizing radiation (Fig. 22 and Fig. 3). We also demonstrated that the (subdominant) component of neutral outflowing circum-galactic hydrogen is primarily comprised of gas that is still undergoing gravitational infall, but has moved past firstpericentric passage, and will subsequently fall back towards the ISM or settle into a approximate rotational equilibrium within the inner CGM (Fig. 2 and Fig. 3). 
We then explore the implications of this scenario for the extended Ly $\alpha$ haloes recently detected around faint $z \sim 3$ Ly $\alpha$ emitting galaxies (LAEs) with the MUSE instrument (Wisotzki et al. 2016. Leclercq et al. 2017). We find that Ly $\alpha$ emitted in-situ within the CGM roughly (but not exactly) follows the radial profile of neutral hydrogen in the simulation (Fig. 3), and traces primarily dense and partially ionized infalling gas, spanning a range of densities and ionization conditions (Fig. 44. We find that angle-averaged emission from the CGM does not vary significantly in time for our simulated galaxy, with variations of a factor of a few at most (Fig. 5). This implies that high-redshift galaxies may be generally surrounded by a diffuse $\operatorname{Ly} \alpha$ halo, irrespective of the $\operatorname{Ly} \alpha$ escaping from the central region.

Combining in-situ emission from the CGM with emission emitted within the central ISM and satellites, and accounting for the MUSE PSF, we find that scattered photons from the central ISM, in-situ emission and emission from satellites contribute comparably to the spatially extended signal (Fig. 6). Scattering dominates the inner arcsec of the Ly $\alpha$ profile, and satellites provide much of the very extended signal (beyond the spatial scales probed by observations of individual LAEs, but which are accessibly via stacking). Extended emission generally last traces inflowing gas before escaping the halo, or otherwise escapes from satellite galaxies without scattering from the CGM. About $60 \%$ of the escaping photons are generated in recombinations, with the remainder generated by collisional excitations (Fig. 7). Compared to a stack of MUSEdetected LAEs from Wisotzki et al. (2018), the time and angleaveraged surface brightness profile from our simulation reproduces the observations to at least 0.2 dex over two orders of magnitude in surface brightness (though admittedly the central arcsec of the profile is severly affected by by PSF-broadening), out to beyond the halo virial radius (Fig. 6, but see also Appendix A).

Finally, we find that the average Ly $\alpha$ spectrum produced by our galaxy is very different from the typical spectrum of an observed LAE (Fig. 8). We find a complex spectral morphology with a dominant peak slightly bluewards of systemic. The total spectrum is composed of a double-peaked spectral component contributed by photons that escape from the ISM without scattering from the CGM, and of a blue single peaked spectral component that traces inflowing circum-galactic gas. This contrasts strongly with the spectral morphology of typical high-redshift LAEs in observations; the spectra of observed LAEs at $z \sim 3$ are usually significantly redshifted from the systemic velocity, are asymmetric with a broad redward tail, and have minimal observed flux bluewards of the systemic velocity (even accounting for IGM attenuation).

This problem seems to apply to other cosmological simulations before IGM attenuation is applied (e.g. Behrens et al. 2019 Smith et al. 2019), but since in our case we analyse emission at redshifts $3<z<4$, IGM attenuation is unlikely to be a sufficient explanation. We speculate that this is indeed a general problem with current simulations (see Section 4 as well as the discussion in Gronke et al. 2017), and that the problems could be related to insufficient resolution in the CGM, and/or because cosmological simulations do not (usually) include cosmic ray creation and transport.

We conclude that observations of spatially resolved Ly $\alpha$ emission from high-redshift galaxies are providing highly challenging constraints for state-of-the-art cosmological simulations. These constraints are particularly timely given the current debate regarding the sensitivity of the phase distribution of the simulated CGM to numerical resolution, and the question of whether cosmic rays play a significant role in shaping the ISM and CGM of galaxies. With the upcoming launch of JWST set to constrain whether high-redshift LAEs are surrounded by extended haloes of Balmer-line emission, it will soon be possible to establish whether in-situ emission from the CGM provides a significant contribution to the extended signal. If confirmed, and given that the extended emission is generally observed to be red-shifted from systemic (Swinbank et al. 2007, Smit et al. 2017, Claeyssens et al. 2019, Leclercq et al. 2020), the implication would then be that the red-shifted spectral morphology of high-redshift Ly $\alpha$ lines is indeed being shaped by a fast-moving and neutral phase of CGM-scale (as opposed to only ISM-scale) galactic outflows with a high covering fraction, a component that is seemingly missing from current state-of-the-art cosmological simulations.

\section{ACKNOWLEDGEMENTS}

We would like to thank Taysun Kimm and Anne Verhamme for providing comments and suggestions that improved the quality of this manuscript. We are grateful to the LABEX Lyon Institute of Origins (ANR-10-LABX-0066) of the Université de Lyon for its financial support within the program "Investissements d'Avenir" (ANR11-IDEX-0007) of the French government operated by the National Research Agency (ANR). This work was supported by Vici grant 639.043.409 from the Netherlands Organisation for Scientific Research (NWO). Simulations were run at the Common Computing Facility (CCF) of LABEX LIO. CC acknowledges support from the Institut Lagrange de Paris and from the European Union Horizon 2020 research and innovation programme under grant agreement No. 818085 GMGalaxies.

\section{DATA AVAILABILITY}

The data underlying this article will be shared on reasonable request to the corresponding author.

\section{REFERENCES}

Ahn S.-H., 2004, ApJ, 601, L25

Aubert D., Pichon C., Colombi S., 2004, MNRAS, 352, 376

Bacon R. et al., 2017, A\&A, 608, A1

Barnes L. A., Haehnelt M. G., Tescari E., Viel M., 2011, MNRAS, 416, 1723

Behrens C., Pallottini A., Ferrara A., Gallerani S., Vallini L., 2019, MNRAS, 486, 2197

Behroozi P. S., Wechsler R. H., Conroy C., 2013, ApJ, 770, 57

Binney J., Tremaine S., 2008, Galactic Dynamics: Second Edition. Princeton University Press

Blondin J. M., Wright E. B., Borkowski K. J., Reynolds S. P., 1998, ApJ, 500, 342

Byrohl C., Gronke M., 2020, A\&A, 642, L16

Cadiou C., Dubois Y., Pichon C., 2019, A\&A, 621, A96

Cantalupo S., Arrigoni-Battaia F., Prochaska J. X., Hennawi J. F., Madau P., 2014, Nature, 506, 63

Cantalupo S., Porciani C., Lilly S. J., 2008, ApJ, 672, 48

Cantalupo S., Porciani C., Lilly S. J., Miniati F., 2005, ApJ, 628, 61

Chisholm J., Tremonti Christy A., Leitherer C., Chen Y., 2016, MNRAS, 463, 541

Chung A. S., Dijkstra M., Ciardi B., Kakiichi K., Naab T., 2019, MNRAS, 484, 2420 
Claeyssens A. et al., 2019, MNRAS, 489, 5022

Dekel A., Birnboim Y., 2006, MNRAS, 368, 2

Díaz C. G., Ryan-Weber E. V., Karman W., Caputi K. I., Salvadori

S., Crighton N. H., Ouchi M., Vanzella E., 2020, MNRAS

Dijkstra M., 2017, arXiv e-prints, arXiv:1704.03416

Dijkstra M., Haiman Z., Spaans M., 2006, ApJ, 649, 14

Dijkstra M., Kramer R., 2012, MNRAS, 424, 1672

Dijkstra M., Lidz A., Wyithe J. S. B., 2007, MNRAS, 377, 1175

Dijkstra M., Loeb A., 2009, MNRAS, 400, 1109

Dubois Y., Volonteri M., Silk J., Devriendt J., Slyz A., Teyssier R., 2015, MNRAS, 452, 1502

Eldridge J. J., Izzard R. G., Tout C. A., 2008, MNRAS, 384, 1109

Eldridge J. J., Stanway E. R., 2016, MNRAS, 462, 3302

Erb D. K., Berg D. A., Auger M. W., Kaplan D. L., Brammer G., Pettini M., 2019, ApJ, 884, 7

Erb D. K., Pettini M., Steidel C. C., Strom A. L., Rudie G. C., Trainor R. F., Shapley A. E., Reddy N. A., 2016, ApJ, 830, 52

Erb D. K., Steidel C. C., Chen Y., 2018, ApJ, 862, L10

Fardal M. A., Katz N., Gardner J. P., Hernquist L., Weinberg D. H., Davé R., 2001, ApJ, 562, 605

Faucher-Giguère C.-A., Kereš D., Dijkstra M., Hernquist L., Zaldarriaga M., 2010, ApJ, 725, 633

Faucher-Giguère C.-A., Lidz A., Zaldarriaga M., Hernquist L., 2009, ApJ, 703, 1416

Federrath C., Klessen R. S., 2012, ApJ, 761, 156

Ferland G. J., Korista K. T., Verner D. A., Ferguson J. W., Kingdon J. B., Verner E. M., 1998, PASP, 110, 761

Fujita A., Martin C. L., Mac Low M.-M., New K. C. B., Weaver R., 2009, ApJ, 698, 693

Furlanetto S. R., Schaye J., Springel V., Hernquist L., 2005, ApJ, 622,7

Gallego S. G. et al., 2018, MNRAS, 475, 3854

Geen S., Rosdahl J., Blaizot J., Devriendt J., Slyz A., 2015, MNRAS, 448, 3248

Genel S., Vogelsberger M., Nelson D., Sijacki D., Springel V., Hernquist L., 2013, MNRAS, 435, 1426

Gentry E. S., Madau P., Krumholz M. R., 2020, MNRAS, 492, 1243

Goerdt T., Dekel A., Sternberg A., Ceverino D., Teyssier R., Primack J. R., 2010, MNRAS, 407, 613

Gronke M., 2017, A\&A, 608, A139

Gronke M., Dijkstra M., McCourt M., Peng Oh S., 2017, A\&A, 607, A71

Gronke M., Girichidis P., Naab T., Walch S., 2018, ApJ, 862, L7

Guillet T., Teyssier R., 2011, Journal of Computational Physics, 230, 4756

Haardt F., Madau P., 1996, ApJ, 461, 20

Hahn O., Abel T., 2011, MNRAS, 415, 2101

Haiman Z., Rees M. J., 2001, ApJ, 556, 87

Hashimoto T. et al., 2017, A\&A, 608, A10

Hashimoto T. et al., 2015, ApJ, 812, 157

Hayes M. et al., 2013, ApJ, 765, L27

Hayes M. J., Runnholm A., Gronke M., Scarlata C., 2020, arXiv e-prints, arXiv:2006.03232

Heckman T. M., Lehnert M. D., Miley G. K., van Breugel W., 1991, ApJ, 381, 373

Heckman T. M., Lehnert M. D., Strickland D. K., Armus L., 2000, ApJS, 129, 493

Hopkins P. F., Kereš D., Oñorbe J., Faucher-Giguère C.-A., Quataert E., Murray N., Bullock J. S., 2014, MNRAS, 445, 581

Hopkins P. F. et al., 2018, MNRAS, 480, 800

Hui L., Gnedin N. Y., 1997, MNRAS, 292, 27
Hummels C. B. et al., 2019, ApJ, 882, 156

Inami H. et al., 2017, A\&A, 608, A2

Inoue A. K., Shimizu I., Iwata I., Tanaka M., 2014, MNRAS, 442, 1805

Keller B. W., Kruijssen J. M. D., 2020, arXiv e-prints, arXiv:2004.03608

Kereš D., Katz N., Weinberg D. H., Davé R., 2005, MNRAS, 363, 2

Kimm T., Blaizot J., Garel T., Michel-Dansac L., Katz H., Rosdahl

J., Verhamme A., Haehnelt M., 2019, MNRAS, 486, 2215

Kimm T., Cen R., 2014, ApJ, 788, 121

Kimm T., Cen R., Devriendt J., Dubois Y., Slyz A., 2015, MNRAS, 451, 2900

Kimm T., Katz H., Haehnelt M., Rosdahl J., Devriendt J., Slyz A., 2017, MNRAS, 466, 4826

Kollmeier J. A., Zheng Z., Davé R., Gould A., Katz N., MiraldaEscudé J., Weinberg D. H., 2010, ApJ, 708, 1048

Kroupa P., 2002, Science, 295, 82

Kulas K. R., Shapley A. E., Kollmeier J. A., Zheng Z., Steidel C. C., Hainline K. N., 2012, ApJ, 745, 33

Lake E., Zheng Z., Cen R., Sadoun R., Momose R., Ouchi M., 2015, ApJ, 806, 46

Laursen P., Razoumov A. O., Sommer-Larsen J., 2009a, ApJ, 696, 853

Laursen P., Sommer-Larsen J., 2007, ApJ, 657, L69

Laursen P., Sommer-Larsen J., Andersen A. C., 2009b, ApJ, 704, 1640

Laursen P., Sommer-Larsen J., Razoumov A. O., 2011, ApJ, 728, 52

Leclercq F. et al., 2020, A\&A, 635, A82

Leclercq F. et al., 2017, A\&A, 608, A8

Levermore C. D., 1984, J. Quant. Spec. Radiat. Transf., 31, 149

Lofthouse E. K. et al., 2019, MNRAS, 2667

Mackenzie R. et al., 2019, MNRAS, 487, 5070

Mas-Ribas L., Dijkstra M., 2016, ApJ, 822, 84

Mas-Ribas L., Dijkstra M., Hennawi J. F., Trenti M., Momose R., Ouchi M., 2017, ApJ, 841, 19

Matsuda Y. et al., 2012, MNRAS, 425, 878

McCourt M., Oh S. P., O'Leary R., Madigan A.-M., 2018, MNRAS, 473, 5407

Michel-Dansac L., Blaizot J., Garel T., Verhamme A., Kimm T., Trebitsch M., 2020, A\&A, 635, A154

Mitchell P. D., Blaizot J., Devriendt J., Kimm T., Michel-Dansac L., Rosdahl J., Slyz A., 2018, MNRAS, 474, 4279

Mori M., Umemura M., Ferrara A., 2004, ApJ, 613, L97

Moster B. P., Naab T., White S. D. M., 2013, MNRAS, 428, 3121

Muzahid S. et al., 2020, MNRAS, 496, 1013

Peeples M. S. et al., 2019, ApJ, 873, 129

Planck Collaboration et al., 2014, A\&A, 571, A16

Rauch M. et al., 2008, ApJ, 681, 856

Reuland M. et al., 2003, ApJ, 592, 755

Rosdahl J., Blaizot J., 2012, MNRAS, 423, 344

Rosdahl J., Blaizot J., Aubert D., Stranex T., Teyssier R., 2013, MNRAS, 436, 2188

Rosdahl J. et al., 2018, MNRAS, 479, 994

Rosdahl J., Teyssier R., 2015, MNRAS, 449, 4380

Rosen A., Bregman J. N., 1995, ApJ, 440, 634

Scannapieco E., 2017, ApJ, 837, 28

Shapley A. E., Steidel C. C., Pettini M., Adelberger K. L., 2003, ApJ, 588, 65

Smit R., Swinbank A. M., Massey R., Richard J., Smail I., Kneib J. P., 2017, MNRAS, 467, 3306 
Smith A., Ma X., Bromm V., Finkelstein S. L., Hopkins P. F., Faucher-Giguère C.-A., Kereš D., 2019, MNRAS, 484, 39

Smith A., Safranek-Shrader C., Bromm V., Milosavljević M., 2015, MNRAS, 449, 4336

Song H., Seon K.-I., Hwang H. S., 2020, ApJ, 901, 41

Stanway E. R., Eldridge J. J., Becker G. D., 2016, MNRAS, 456, 485

Steidel C. C., Bogosavljević M., Shapley A. E., Kollmeier J. A., Reddy N. A., Erb D. K., Pettini M., 2011, ApJ, 736, 160

Steidel C. C., Erb D. K., Shapley A. E., Pettini M., Reddy N., Bogosavljević M., Rudie G. C., Rakic O., 2010, ApJ, 717, 289

Suresh J., Nelson D., Genel S., Rubin K. H. R., Hernquist L., 2019, MNRAS, 483, 4040

Swinbank A. M., Bower R. G., Smith G. P., Wilman R. J., Smail I., Ellis R. S., Morris S. L., Kneib J. P., 2007, MNRAS, 376, 479

Tapken C., Appenzeller I., Noll S., Richling S., Heidt J., Meinköhn E., Mehlert D., 2007, A\&A, 467, 63

Tasitsiomi A., 2006, ApJ, 645, 792

Teyssier R., 2002, A\&A, 385, 337

Thornton K., Gaudlitz M., Janka H. T., Steinmetz M., 1998, ApJ, 500, 95

Trainor R. F., Steidel C. C., Strom A. L., Rudie G. C., 2015, ApJ, 809, 89

Trebitsch M., Blaizot J., Rosdahl J., Devriendt J., Slyz A., 2017, MNRAS, 470, 224

Tweed D., Devriendt J., Blaizot J., Colombi S., Slyz A., 2009, A\&A, 506, 647

van de Voort F., Springel V., Mandelker N., van den Bosch F. C., Pakmor R., 2019, MNRAS, 482, L85

Verhamme A., Dubois Y., Blaizot J., Garel T., Bacon R., Devriendt J., Guiderdoni B., Slyz A., 2012, A\&A, 546, A111

Verhamme A. et al., 2018, MNRAS, 478, L60

Verhamme A., Schaerer D., Atek H., Tapken C., 2008, A\&A, 491, 89

Verhamme A., Schaerer D., Maselli A., 2006, A\&A, 460, 397

Wang B., 1995, ApJ, 444, 590

Wisotzki L. et al., 2016, A\&A, 587, A98

Wisotzki L. et al., 2018, Nature, 562, 229

Wofford A., Leitherer C., Salzer J., 2013, ApJ, 765, 118

Yusef-Zadeh F., Morris M., White R. L., 1984, ApJ, 278, 186

Zheng Z., Cen R., Trac H., Miralda-Escudé J., 2010, ApJ, 716, 574

Zheng Z., Cen R., Weinberg D., Trac H., Miralda-Escudé J., 2011, ApJ, 739, 62

Zheng Z., Miralda-Escudé J., 2002, ApJ, 578, 33

\section{APPENDIX A: COMPARING TO STACKED Ly $\alpha$ SURFACE BRIGHTNESS PROFILES FROM MUSE OBSERVATIONS}

In Section 3.4 we compared a mean-stacked and angle-averaged surface brightness profile of our simulated galaxy with a stack of observed LAEs from Wisotzki et al. (2018). In Fig. A1, we perform a more realistic comparison with the observations by median stacking (after first azimuthal averaging), by using mock data cubes orientated along 12 random lines of sight for each simulation output (rather than angle averaging), and by applying a flux cut to mimic the sensitivity of MUSE in detecting LAEs. We choose a flux cut of $\log _{10}\left(F_{\mathrm{Ly} \alpha} / \mathrm{ergs}^{-1} \mathrm{~cm}^{-2}\right)>-17.6$ in order to match the peak surface brightness of the observed stack, which is comparable to the approximate flux limit for which the MUSE observations are complete (see, e.g., figure 1 in Leclercq et al. 2017). With this flux

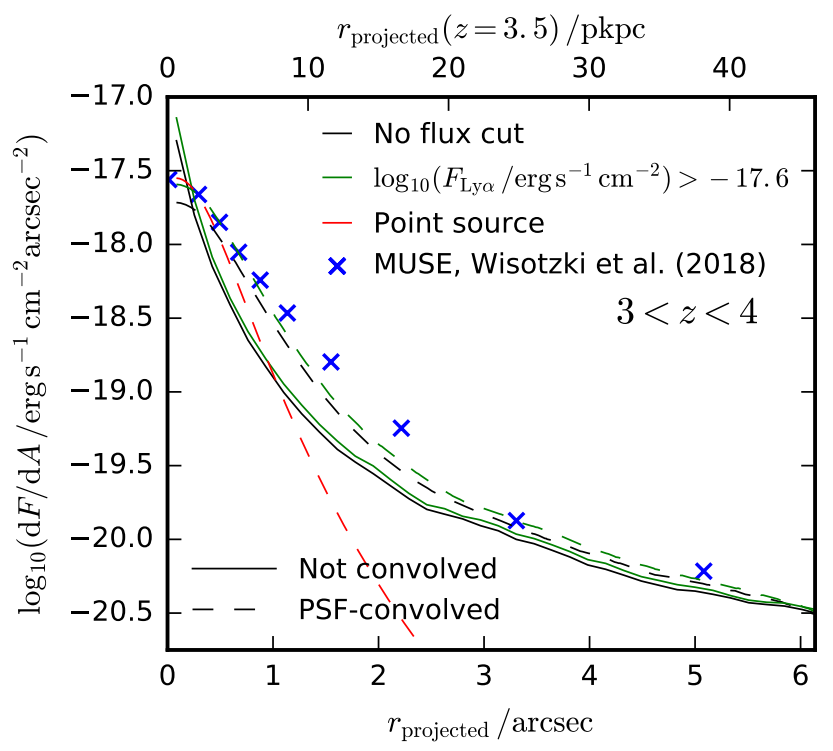

Figure A1. A comparison of the predicted Ly $\alpha$ surface brightness profile from our simulation (for $3<z<4$ ) compared to the stack of LAEs produced using deep MUSE data by Wisotzki et al. (2018). In this case we more closely reproduce the observational selection and methodology by median stacking (after azimuthal averaging), by using mock data cubes orientated along random lines of sight (rather than angle averaging) and by applying a flux cut to mimic the sensitivity of MUSE in detecting LAEs. Solid (dashed) lines show profiles without (with) convolution with the appropriate MUSE point spread function (PSF). The red dashed line shows the convolved profile of a point source for comparison, scaled to match the total luminosity of the simulation stack when no flux cut is applied. We choose a flux cut of $\log _{10}\left(F_{\text {Ly } \alpha} / \operatorname{ergs}^{-1} \mathrm{~cm}^{-2}\right)>-17.6$ in order to match the peak surface brightness of the observed stack, which is comparable to the approximate flux limit for which the MUSE observations are complete (see, e.g., figure 1 in Leclercq et al. 2017). With this flux cut, we reproduce the observed profile to within at least 0.2 dex over the plotted range.

cut, we reproduce the observed profile to within at least $0.2 \mathrm{dex}$ over the plotted range.

The mock data cubes used here make use of the peeling off technique in order to obtain sufficient signal along specific lines of sight (which in turn is needed to apply an observational flux cut). By using peeling off, we lose the information about the origin of each individual photon, which is why we do not use the mock cubes for the results presented in Sec 3 With the adopted flux cut, the simulated surface brightness profile in any case very closely resembles the mean, angle-averaged stack shown in Fig. 6 